\title{
A Study on Dual Readout Crystal Calorimeter for Hadron and Jet Energy Measurement at a future Lepton Collider
}

\author{
G.P. Yeh, Fermilab \\ (Dated: January 11, 2010)
}

\begin{abstract}
Studies of requirements and specifications of crystals are necessary to develop a new generation of crystals for dual readout crystal hadron or total absorption calorimeter. This is a short and basic study of the characteristics and hadron energy measurement of PbWO4 and BGO crystals for scintillation and Cerenkov Dual Readout hadron calorimeter.
\end{abstract}

PACS numbers:

\section{INTRODUCTION}

Scintillation and Cerenkov Dual Readout Calorimeters are excellent candidates for the calorimeters to have the high precision of $30 \% / \sqrt{E}$ for hadron jet energy measurement required for the future large lepton colliders Muon Collider, CLIC, ILC [1-6]. L3[7] used BGO, and CMS[8] is using PbWO4 for their EM calorimeters. Scintillation and Cerenkov Dual Readout using BGO, PbWO4, and various crystals as EM calorimeter in combination with the Dual Readout fiber Hadron Calorimeter have been studied extensively with test beam data and simulation studies $[1,2,4]$. Feasibility studies are being performed for the proposed Total Absorption Crystal Calorimeter $[9,10]$ to use crystal[11] for both the EM calorimeter and the Hadron calorimeter for measuring hadron and jet energy.

The main questions on Dual Readout Crystal Total Absorption or Hadron calorimeter are:

- Requirements and Specifications[12, 13] for producing affordable crystals BGO cost $[12,13]$ is $\$ 10$ per $\mathrm{cm}^{3}$. The cost for a $100 \mathrm{~m}^{3}$ BGO calorimeter would be a prohibitive $\$ 1$ billion. As Alexander Gektin summarized on Oct. 27, 2009 in the Fermilab Research Techniques Seminar,[12] criteria and specifications are necessary to develop a new generation of crystals for Total Absorption Crystal calorimeter. $\mathrm{PbWO} 4[8]$ with a cost of $\$ 3$ per $\mathrm{cm}^{3}$ is more affordable than BGO. The cost of crystal for dual readout hadron calorimeter should be $\approx \$ 1$ per $\mathrm{cm}^{3}$.

- Interaction lengths and Leakage

Studies based on data from 1980s experiments have shown that 8 interaction lengths, needed to contain $95 \%$ of $300 \mathrm{GeV}$ pions, are required for CLIC[14] and/or Muon Collider[3] calorimeters. CsI and other lower density crystals would not have the required interaction lengths. For the same detector geometry and size, $\mathrm{PbWO} 4$ would have more interaction lengths for more containment of hadron showers than BGO. With the CCAL02 geometry in the present simulation, the EM + HA Crystal Calorimeter BGO has 5.8 interaction lengths while $\mathrm{PbWO} 4$ has 7 interaction lengths. Alternatively, for the same number of interaction lengths, crystals such as $\mathrm{PbWO} 4$ with higher density than BGO, would enable having smaller radius for the calorimeter and the solenoid.

- Crystal characteristics

The light yields, which may be an important consideration for energy measurement, are relatively $\approx 50: 1$ for $\mathrm{BGO}$ and $\mathrm{PbWO}$. The signal decay time is $300 \mathrm{~ns}$ for $\mathrm{BGO}$ and $30 \mathrm{~ns}$ for PbWO4. The crystal to be developed for dual readout hadron calorimeter must enable excellent measurement of Cerenkov and scintillation signals.

- Segmentation and Calibration

The calorimeter segmentation and necessary calibration will be a major issue and will need extensive study, which will require digitization and simulation of the photon detection efficiency and calibration of scintillation and Cerenkov optical readout, for example, with the Silicon Photomultiplier, SiPM.

- Detector simulation Physics Models

Geant4 and FLUKA simulation software are commonly used. The models/parameters QGSC, QGSP, QGSP_BERT, LHEP, LCPHYS in GEANT4 simulate different detector responses. BGO calorimeter response for the above physics models have been reported by Hans Wenzel at ALCPG09 Workshop [6]. Test Beam data 
is needed for Validation of Simulations. A study using charged tracks, to correct for the effects of the magnetific field for Jet and Jet-Jet mass reconstruction, has also been reported by Adam Para at ALCPG09 Workshop.[6]

Feasibility studies of dual readout crystal calorimeter will require simulation studies similar to this study, with crystals of various characteristics, to develop new types of crystals. In this short study, we compare the hadron energy measurement for $\mathrm{BGO}$ and $\mathrm{PbWO} 4$ dual readout crystal calorimeter, for 2 sizes of segmentation.

Simulation is briefly described in Section II. Analysis is outlined in Section III. Results are shown in Section IV. The Summary is in Section V.

Additional plots are also provided in Appendix A. Some of Hans Wenzel's results [5] from helping this study are in Appendix B. Introductory Computing info are listed in Appendix C. Dual Readout Crystal Calorimeter analysis framework is given in Appendix D, The necessary modifications from BGO Simulation to PbWO4 Simulation are listed in Appendix E. Information and example for using Open Science Grid (OSG) are listed in Appendix F.

\section{SIMULATION}

The two main types of particles simulated are:

- Single particles, electrons, pions at various energies (Table I)

to provide $\mathrm{C} / \mathrm{S}$ calibration, energy containment, and energy resolution studies.

- Single $W$ and single $Z$, with momentum 50,100, $200 \mathrm{GeV} / \mathrm{c}$, for studying di-jet mass resolution.

\begin{tabular}{l|cccccccc}
\hline \hline Single particles: & & & & & & & & \\
Energy $(\mathrm{GeV}):$ & $\pi^{-}$ & 1 & 5 & 10 & 20 & 50 & 100 & 200 \\
& $e^{-}$ & & & 10 & & 50 & & \\
\hline \hline
\end{tabular}

TABLE I: Pion and $e^{-}$Single particle Energies

The main Calorimetry characteristics are: Response, Linearity, Containment, Shower shape, and Energy Resolution. The dual readout crystal calorimeter feasibility studies must have comparisons of various crystals. Because of this study, PbWO4 Crystal detector type was created

/ilc/ild/wenzel/ccal02/ccal02_PbWO4 and /ilc/ild/gpyeh/ccal02/ccal02_PbWO4

To study Interaction Lengths and Energy Leakage, we also created "PbWO4_d15" (with density = 15) Crystal detector /ilc/ild/gpyeh/ccal02/ccal02_PbWO4_d15

Also because of this study, Adam Para and Hans Wenzel wrote a script to enable creating crystal detectors with different density and/or different transverse segmentation. We study $\pi^{-}$energy resolution for BGO and PbWO4 Crystals, and for density $=10,15,20 \mathrm{~g} / \mathrm{cm}^{3}$.

In the CCAL02 simulation, the detector segmentation (Table II) has been: 


\begin{tabular}{r|c|c|c|c|c|c|c}
\hline \hline & Layers & $\begin{array}{c}\text { length/layer } \\
\mathrm{cm}\end{array}$ & $\begin{array}{c}\text { depth } \\
\mathrm{cm}\end{array}$ & $\begin{array}{c}\text { Segmentation } \\
\mathrm{cm} \times \mathrm{cm}\end{array}$ & $\begin{array}{c}\text { inner radius } \\
\mathrm{cm}\end{array}$ & $\begin{array}{c}\text { outer radius } \\
\mathrm{cm}\end{array}$ & $\begin{array}{c}\text { inner z max } \\
\mathrm{cm}\end{array}$ \\
\hline Ecal Barrel & 8 & 3 & 24 & $3 \times 3$ & 127 & 151 & 192 \\
Hcal Barrel & 17 & 6 & 102 & $5 \times 5$ & 151 & 253 & 294 \\
Total Barrel & 25 & & 126 & & & & \\
\hline \hline Ecal Endcap & 8 & 3 & 24 & $3 \times 3$ & & & \\
Hcal Endcap & 17 & 6 & 102 & $5 \times 5$ & & & \\
Total Endcap & 25 & & 126 & & & & \\
\hline \hline
\end{tabular}

TABLE II: Crystal Calorimetry Segmentation. BGO and PbWO4 crystals have 5.8 and 7 Interaction lengths, respectively. We use $1.5 \times 1.5 \times 3$ for the second Ecal segmentation size, and 2.5x2.5x6 for the second Hcal segmentation size.

In the detector description/geometry: Barrel EM calorimeter radius $=24$ (127 to 151) cm within $\mathrm{z}= \pm 192 \mathrm{~cm}$; HAD calorimeter radius $=102(151$ to 253$) \mathrm{cm}$, within $\mathrm{z}= \pm 294 \mathrm{~cm}$; Solenoid (5 Tesla) starts at $255 \mathrm{~cm}$ radius, within $\mathrm{z}= \pm 288 \mathrm{~cm}$. The CCAL02 crystal calorimeter simulation has no gaps longitudinally between crystals.

The L3 BGO [7] and CMS PbWO4 [8] Electromagnetic calorimeters both have truncated-pyramid-shaped crystals arranged in the projective geometry, minimizing transverse and longitudinal gaps in the calorimeter. In the future, more realistic geometry of the CCAL02 calorimeter, including shapes and gaps, should be implemented for the Crystal Calorimeter simulation studies.

Studying the longitudinal segmentation will be important. Varying longitudinal segmentation is not yet implemented in the CCAL02 simulation. In the future, the simulation should have:

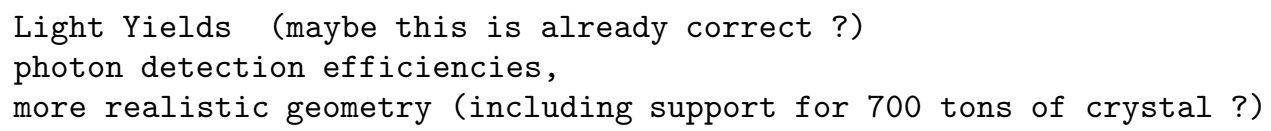

These can take quite some time to implement. Segmentation studies would be more meaningful when we have the above. Mark Fischler mentioned also the effect of the angle of the light. Calibration is an important issue for calorimetry. Experimentalists know that these take lots of work and time. Just getting the correct "dual readout correction" (Over-correction? Energy dependence ? as indicated in Appendix B) seems challenging and may need quite some time.

For the Crystal Dual Readout calorimeter CCAL02 simulation studies including this one, the Simulation tools (Appendix C, D, E, F) are: SLIC simulation environment, GEANT4 LCPHYS physics list, Optical calorimeter by Hans Wenzel, lcsim.org jas3 analysis framework. The simulation, computing, and analysis resources are available on ilcsim.fnal.gov and ilcsim2.fnal.gov clusters. Larger samples are produced on FermiGrid and Open Science Grid (OSG). 


\section{ANALYSIS}

Each high energy hadron interaction produces secondary particles. On average, about $1 / 3$ of the secondary particles are neutral pions which quickly decay into 2 photons producing subsequent electromagnetic showers. The other hadronic secondary particles are mostly charged pions and deposit energy in the calorimeter through $\mathrm{dE} / \mathrm{dx}$ ionization or interacts hadronicly and create subsequent showers. The ratio of neutral to charged, the em fraction f_em, increases with hadron energy, and causes non-linear hadronic energy response of calorimeters. The large fluctuation in f_em, dominantly causes the poor energy resolution from the traditional calorimetry based on scintillation signal alone. A calorimeter with Cerenkov (C) and Scintillation (S) dual readout enables the direct measurement of both the electromagnetic neutral component and the charged component for each hadron shower, thus provide significantly improvement in the precision of the hadron or jet energy measurement.

The 2nd largest fluctuation fluctuation is the missing energy due to nuclear breakup. Measuring also the slow neutrons from the hadron shower could further imrpove the hadron energy measurement.

The calibration of the calorimeter scintillation and Cerenkov response to be achieved with electrons and pions with the same energy, to measure the e/h ratio, which is an important characteristic of each calorimeter, calibrating $\left(\frac{e}{h}\right)_{C}$ and $\left(\frac{e}{h}\right)_{S}$. For each hadron, the C/S ratio provides a measurement of f_em. For hadrons with the same energy, the scintillation response relates linearly with f_em. Energy measurement is improved by correcting the scintillation response with $\mathrm{C} / \mathrm{S}$ and $\mathrm{e} / \mathrm{h}$.

For the crystal density and hadron shower leakage study, the first and most important measurement is the raw Scintillation energy deposition, for incident charged pions at different energies (e.g. 10, 20, 50, 100 GeV), for crystals of different densities or with different interaction lengths.

Calibration for the crystal calorimeter is being done by establish calibration factors for $\mathrm{C}$ and $\mathrm{S}$ signals using 10 $\mathrm{GeV}$ electrons, and calibrating the response of hadrons using $10 \mathrm{GeV}$ pions. The scintillation energy deposition for $10 \mathrm{GeV} e^{-}$for BGO and PbWO4 is shown in Figure 1. Calibration should probably be done using $50 \mathrm{GeV}$ electrons, and calibrate response of hadrons using $50 \mathrm{GeV}$ pions. The $Z \rightarrow e^{+} e^{-}$events would be the events for calibration in a real detector, and are excellent for the Monte Carlo simulations. Examples of Cerenkov and scintillation response for $10 \mathrm{GeV}$ and $50 \mathrm{GeV} e^{-}$are shown in Figure 17.
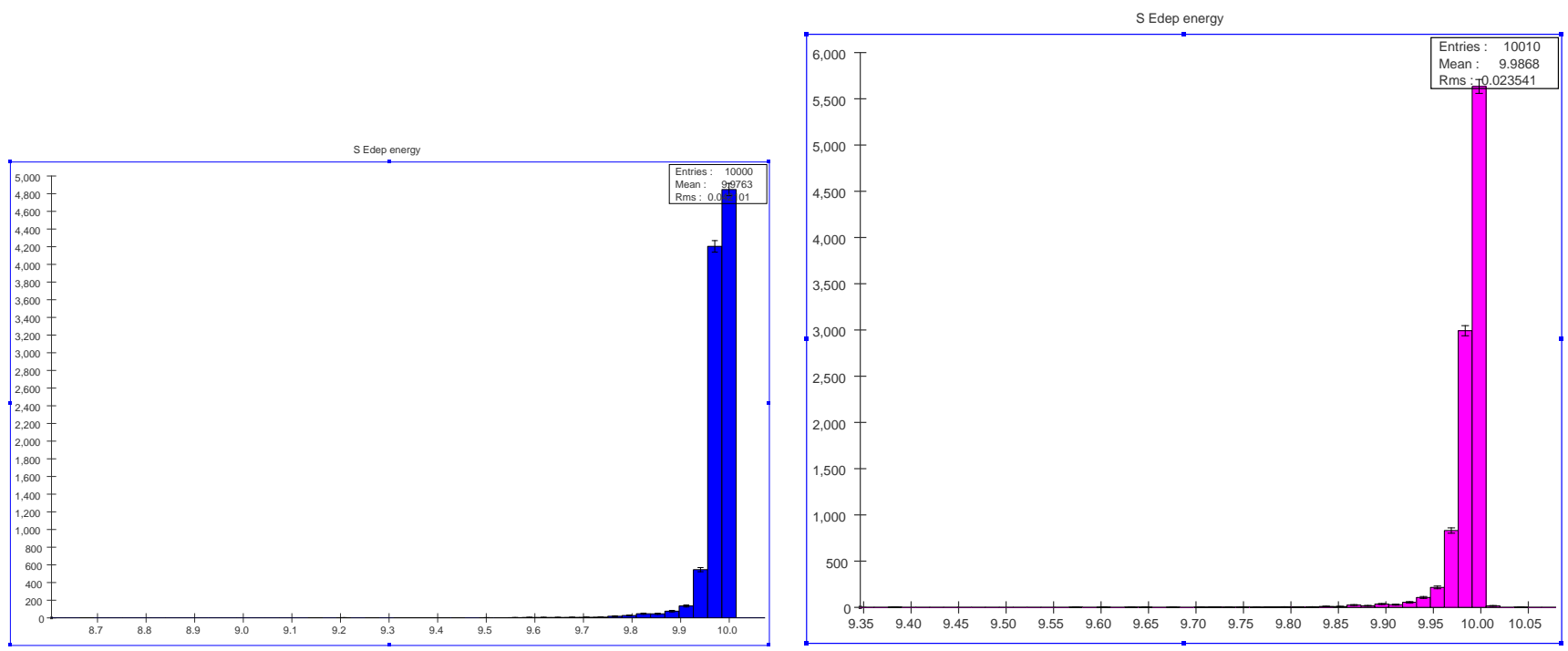

FIG. 1: LCPhys BGO and PbWO4 scintillation energy deposition for $10 \mathrm{GeV} e^{-}$ 


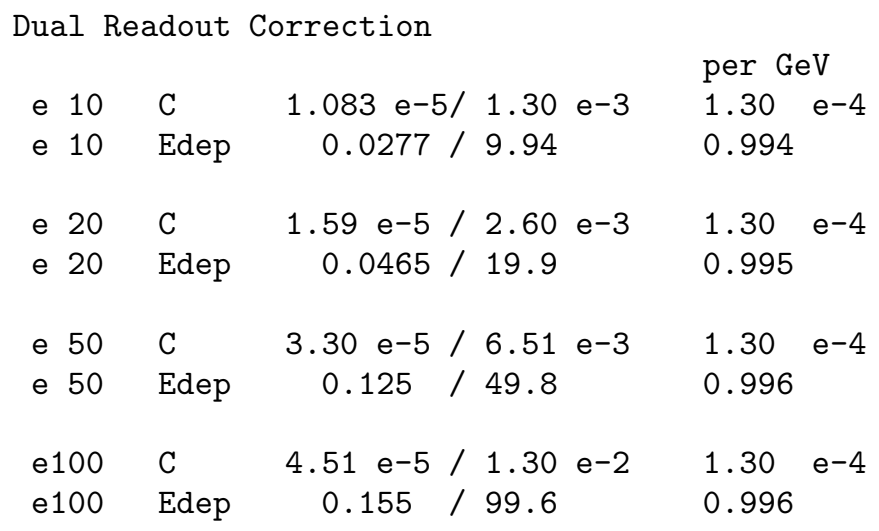

For the crystal density and hadron shower leakage study, the first and most important measurement is the raw Scintillation energy deposition, for incident charged pions at different energies (e.g. 10, 20,50, $100 \mathrm{GeV}$ ), for crystals of different densities or with different interaction lengths.

Energy deposition and dual readout corrected energy in BGO, PbWO4, d15 are shown for $20 \mathrm{GeV} \pi^{-}$in Figure 2, and for $100 \mathrm{GeV} \pi^{-}$in Figure 3. Compared with the Energy depostion as determined from the scintillation signal alone, the dual readout corrected energy has improved energy resolution, as observed in these Figures and also summarized in Table III. The "d15" crystal with density $=15 \mathrm{~g} / \mathrm{cm}^{3}$ has better energy resolution than BGO or $\mathrm{PbWO} 4$, which have fewer interaction lengths due to their lower density.

Cerenkov signal C vs. Scintillation signal $\mathrm{S}$ for $100 \mathrm{GeV} \pi^{-}$in BGO, PbWO4, d15, d20 are shown in Figure 4. The signal leakage is evident for BGO with 5.8 interaction lengths or PbWO4 with 7 interaction lengths.

Energy deposition $\mathrm{S}$ in BGO, PbWO4, d15, d20 for $200 \mathrm{GeV} \pi^{-}$are shown in Figure 5. The significant energy leakage is observed for BGO with 5.8 interaction lengths or PbWO4 with 7 interaction lengths. 


\section{A. Dual Readout Correction}

Cerenkov and Scintillation Dual Readout calorimetry improve the hadron energy resolution, as have been demonstrated by the DREAM and 4th Collaborations, $[1,2,4,6]$ and by the recent CCAL02 studies. $[5,6]$ The improvement in hadron energy resolution because of Scintillation and Cerenkov Dual Readout is observed in Table III and Figure 6 .

The dual readout correction to the scintillation signal depends on the $\mathrm{C} / \mathrm{S}$ calibration for the characteristics (e.g. Light Yield, density) of the calorimeter, and likely also the geometry including segmentation of the calorimeter. Meaningful study of Dual Readout correction would requite more realistic simulation than the present CCAL02.

For the present CCAL02 studies, Adam Para uses QGSP_BERT in GEANT4.

The scintillation and Cerenkov signals are calibrated using $10 \mathrm{GeV}$ electrons, with Mean_S_Edep $=9.9845$ and mean_Cheren $=0.0015304$ and the Dual Readout correction Scorrected $=S /(0.681+0.311 \times C / S)$ is applied to the scintillation signal to improve energy measurement.

Hans Wenzel usually uses LCPhys in GEANT4, with various Dual Readout corrections. Examples of Hans' Dual Readout corrections and results are shown in Appendix B. After looking into comparisons of different crystal densities (BGO, PbWO4, d15) and hadron shower leakage, Hans is considering energy dependent corrections.

For the Muon Collider, the calorimeter should have at least 8 interaction lengths (see Figure 14). The Test Beam dual readout hadron or total absorption crystal calorimeter should have $\geq 10$ interaction lengths, large enough to contain $>95 \%$ of $100 \mathrm{GeV}$ charged pions. 

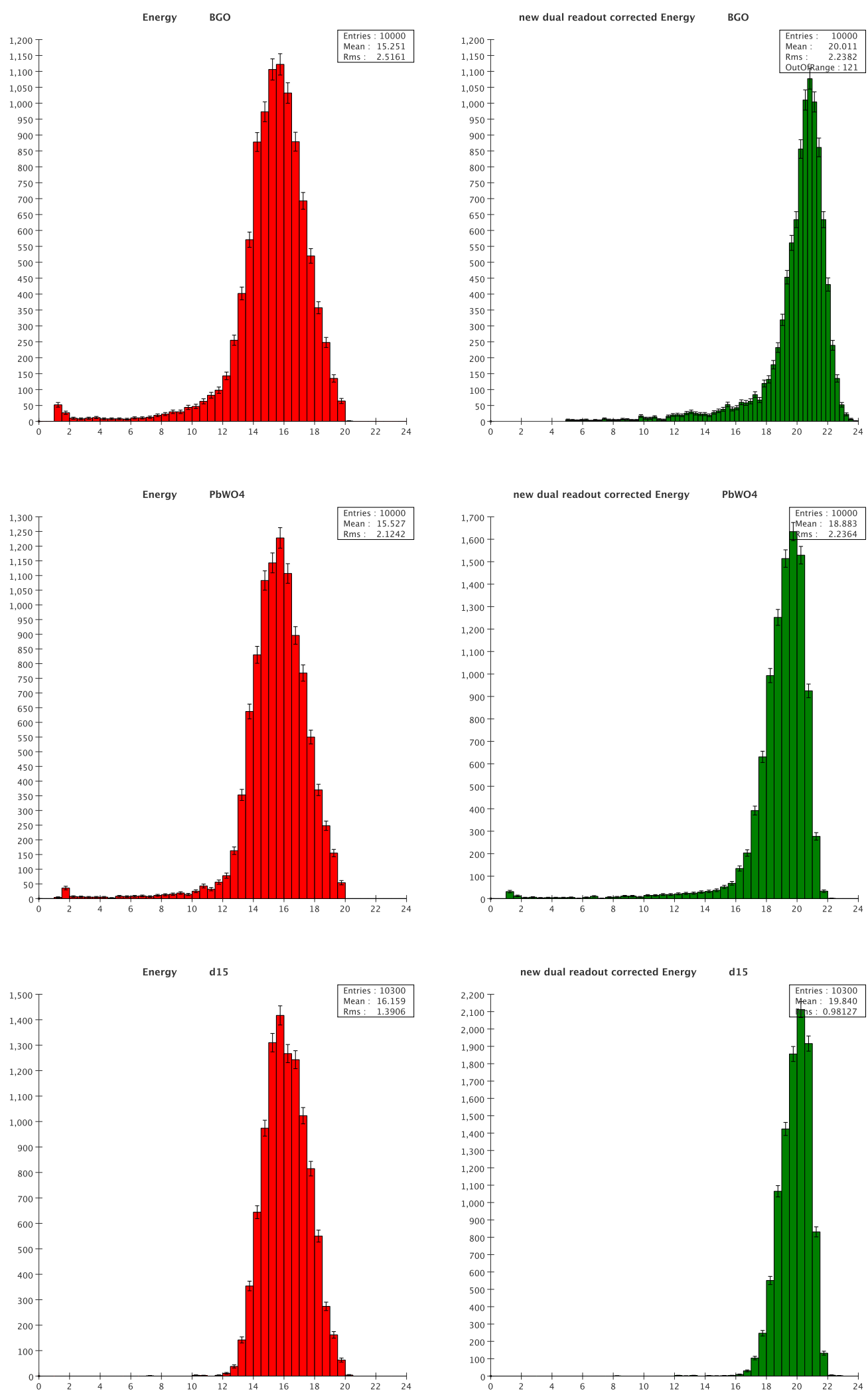

FIG. 2: Energy deposition (left) and dual readout corrected energy (right) for $20 \mathrm{GeV} \pi^{-}$in BGO, PbWO4, D15 

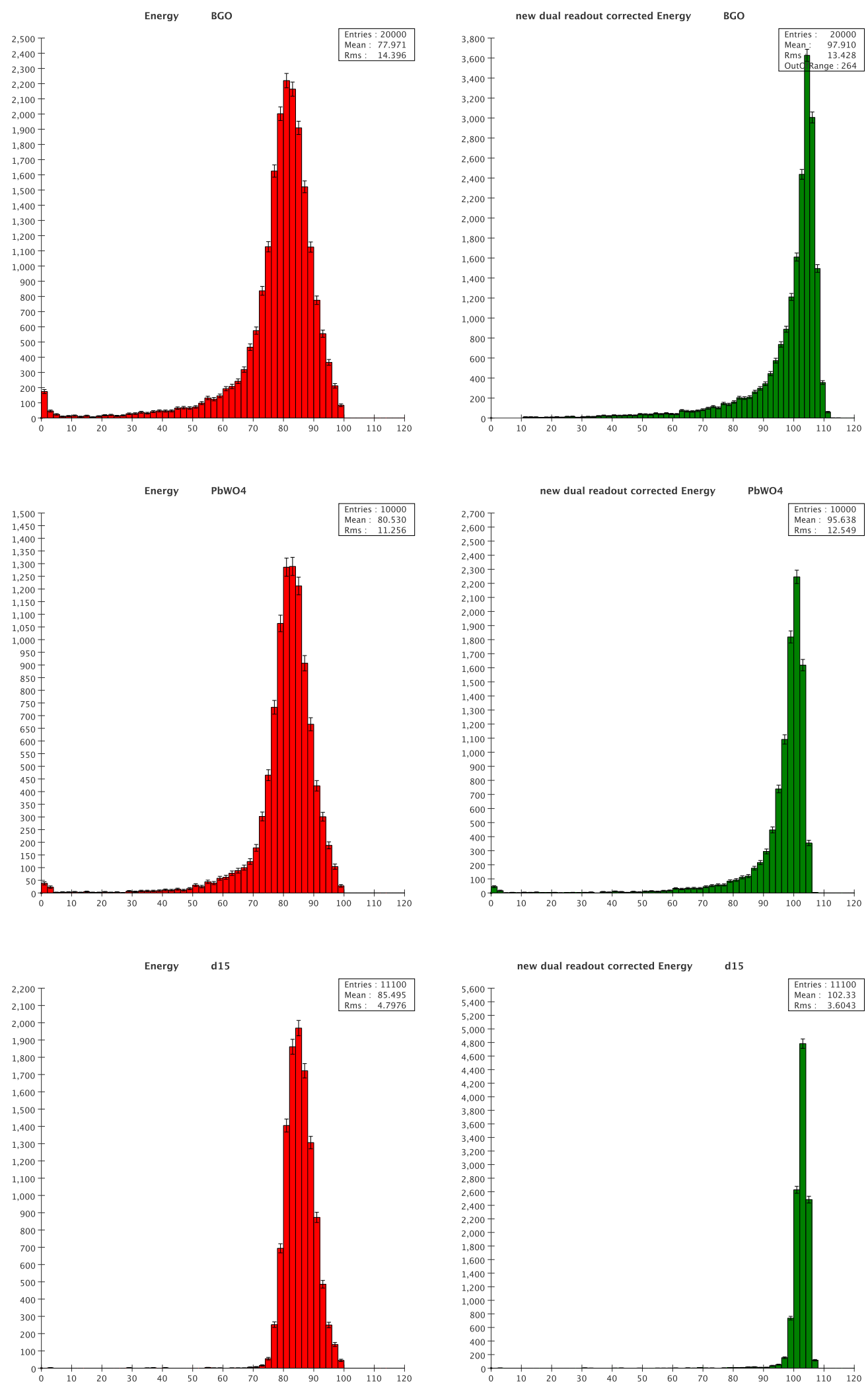

FIG. 3: Energy deposition (left) and dual readout corrected energy (right) for $100 \mathrm{GeV} \pi^{-}$in $\mathrm{BGO}, \mathrm{PbWO} 4, \mathrm{~d} 15$ 

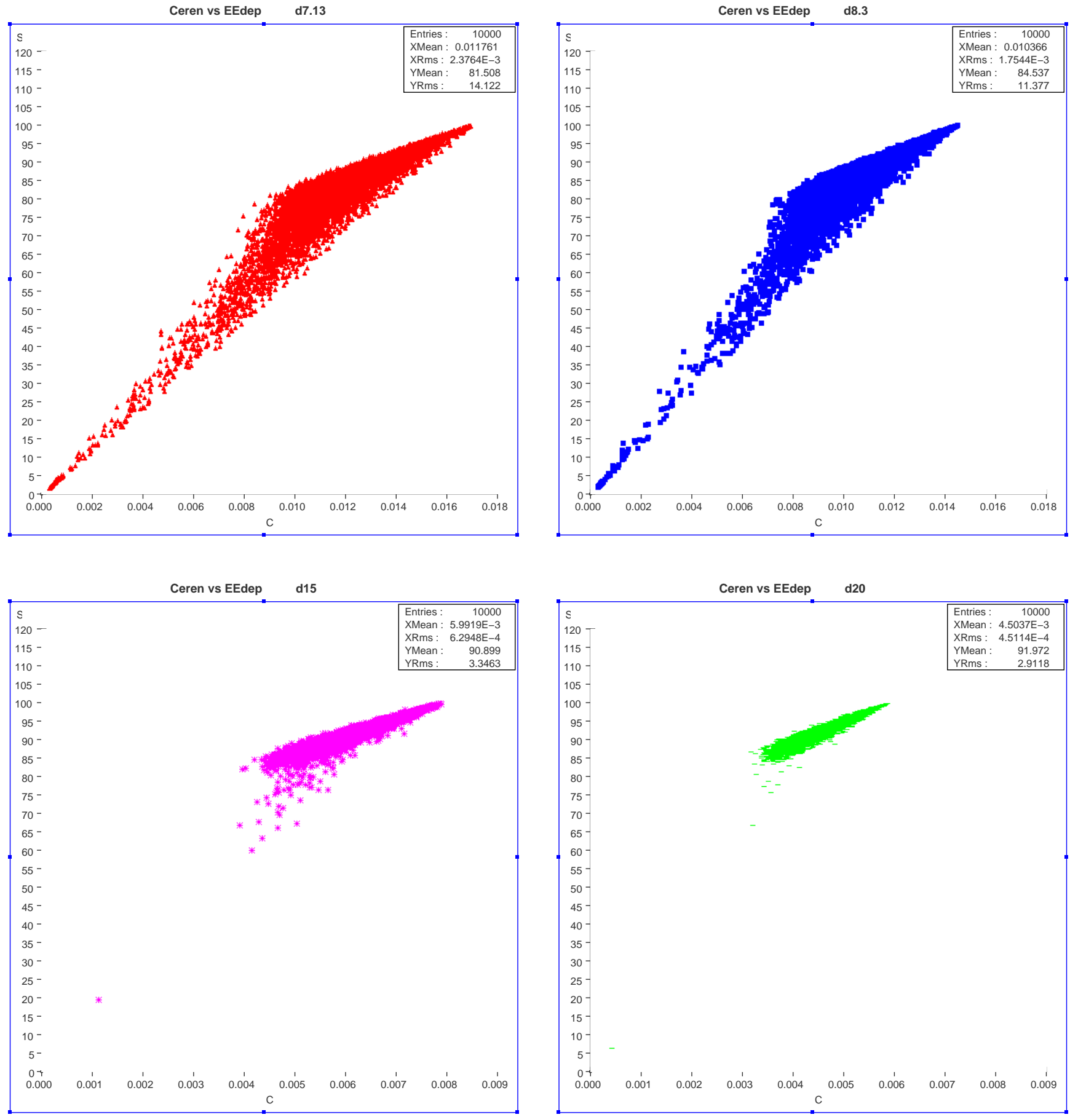

FIG. 4: $\mathrm{C}$ vs. $\mathrm{S}$ for $100 \mathrm{GeV} \pi^{-}$in BGO, PbWO4, d15, d20 

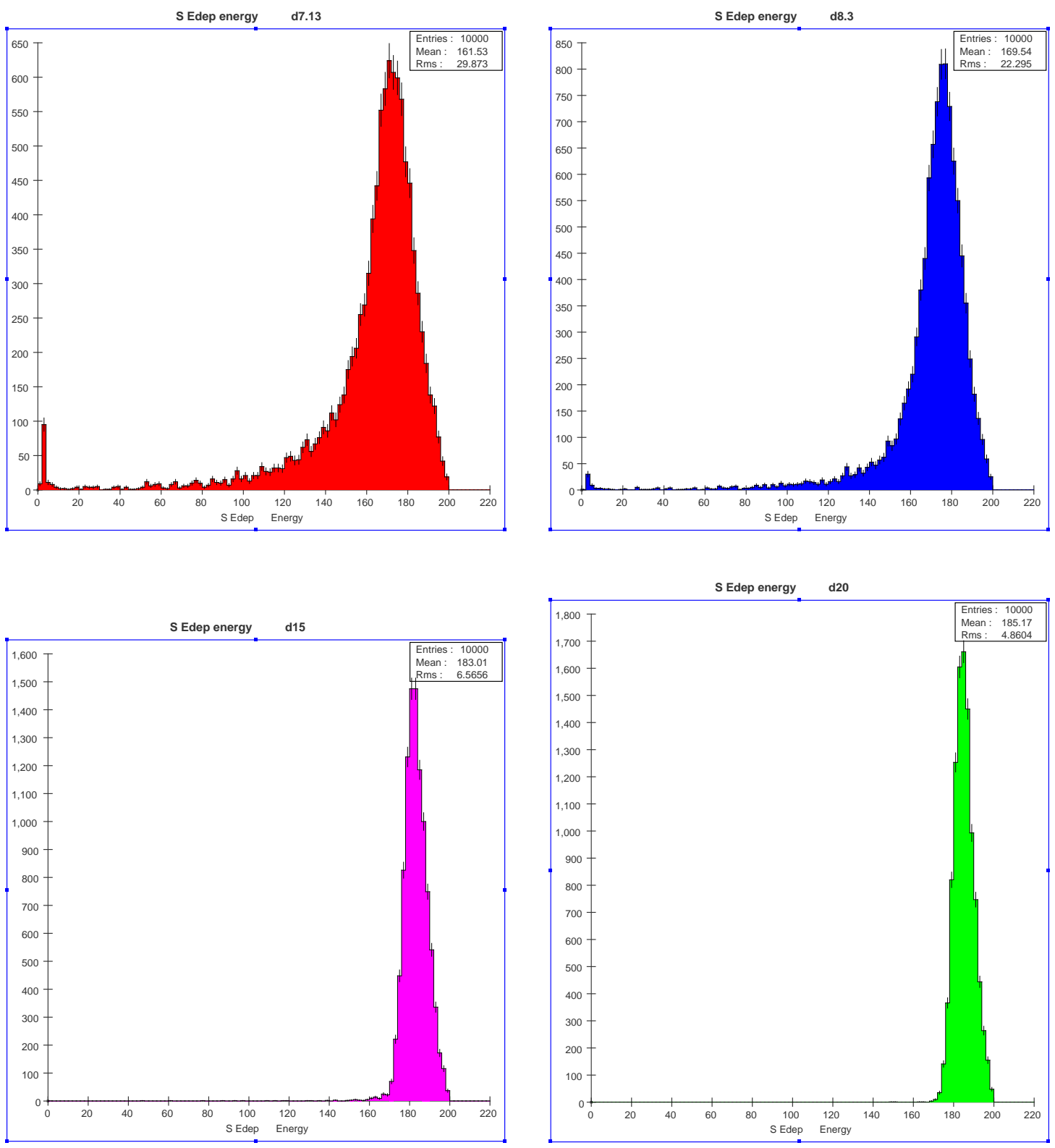

FIG. 5: $200 \mathrm{GeV} \pi^{-}$energy deposition, Edep, in BGO, PbWO4, d15, d20 


\section{RESULTS}

\section{A. Interaction Lengths and Leakage}

The rms/mean vs E before and after Dual Readout C/S Correction for crystal density = 7.13 BGO, 8.3 PbWO4, d15. are shown in Table III and in Figure 6.

The fraction of energy observed, Edep(S) / E, and rms/mean of (Energy deposition, Edep (S)), in BGO, PbWO4, d15, d20 for $\pi^{-}$with 10, 20, 50, 100, or $200 \mathrm{GeV}$ energy are shown in Table IV and Figure 7.

In this brief simulation comparison study, hadron shower leakage is clearly observed in Figures $2,3,4,5,6,7$ and in Table III and Table IV for BGO with 5.8 interaction lengths and PbWO4 with $7 \lambda_{I}$, but not for d15 and d20 crystals with densities of $15 \mathrm{~g} / \mathrm{cm}^{3}$ or $20 \mathrm{~g} / \mathrm{cm}^{3}$. With 5.8 or 7 interaction lengths, the hadron shower leakage significantly degrades the hadron energy resolution before and/or after dual readout correction.

The hadron shower leakage, as shown in APPENDIX B, has also been observed [5] and confirmed by Hans Wenzel after the start of this short study. Data from previous experiments and studies indicate that 8 interaction lengths to contain $95 \%$ of $300 \mathrm{GeV}$ pion.[14]

In addition to this short study with varialble crystal density, other ways to study hadron shower containment and leakage could be:

1) varying the thickness of the hadron calorimeter in CCAL02 (may also need to change the radius and magnetic field of the solenoid)

2) simulation of a Test-Beam-like test detector with variable hadron calorimeter thickness. . 


\begin{tabular}{l|c|c|c|c|c|c|c|c|c}
\hline \hline Crystal & $\begin{array}{c}\text { Density } \\
{\left[\mathrm{g} / \mathrm{cm}^{3}\right]}\end{array}$ & $\begin{array}{c}\mathrm{X}_{0} \\
{[\mathrm{~cm}]}\end{array}$ & $\begin{array}{c}\lambda_{I} \\
{[\mathrm{~cm}]}\end{array}$ & $\begin{array}{c}\mathrm{N} \\
{\left[\lambda_{I}\right]}\end{array}$ & $\begin{array}{c}10 \mathrm{GeV} \pi^{-} \\
\mathrm{rms} / \text { mean }\end{array}$ & $\begin{array}{c}20 \mathrm{GeV} \pi^{-} \\
\mathrm{rms} / \mathrm{mean}\end{array}$ & $\begin{array}{c}50 \mathrm{GeV} \pi^{-} \\
\mathrm{rms} / \text { mean }\end{array}$ & $\begin{array}{c}100 \mathrm{GeV} \pi^{-} \\
\mathrm{rms} / \text { mean }\end{array}$ & $\begin{array}{c}200 \mathrm{GeV} \pi^{-} \\
\mathrm{rms} / \mathrm{mean}\end{array}$ \\
\hline LCPhys BGO & 7.13 & 1.12 & 21.88 & 5.8 & $1.1 / 7.1$ & $2.5 / 15.3$ & $6.7 / 39.0$ & $14.4 / 78.0$ & $29.9 / 155.2$ \\
& & & & & $1.0 / 9.9$ & $2.2 / 20.0$ & $6.0 / 49.9$ & $13.4 / 97.9$ & $26.1 / 166.7$ \\
\hline LCPhys PbWO4 & 8.3 & 0.9 & 18 & 7 & $1.0 / 7.2$ & $2.1 / 15.5$ & $5.5 / 39.9$ & $11.3 / 80.5$ & $23.9 / 161.4$ \\
& & & & & $0.9 / 9.9$ & $1.7 / 20.3$ & $4.6 / 51.2$ & $9.6 / 101.6$ & $23.1 / 199.2$ \\
\hline LCPhys d15 & 15 & & & & $0.82 / 7.5$ & $1.4 / 16.2$ & $2.7 / 42.1$ & $4.8 / 85.5$ & \\
& & & & & $0.70 / 9.5$ & $1.0 / 19.8$ & $1.5 / 51.0$ & $3.6 / 102.3$ & \\
\hline \hline
\end{tabular}

\begin{tabular}{l|l|c|c|c|c|c|c|c|c}
\hline \hline Crystal & $\begin{array}{c}\text { Density } \\
{\left[\mathrm{g} / \mathrm{cm}^{3}\right]}\end{array}$ & $\begin{array}{c}\mathrm{X}_{0} \\
{[\mathrm{~cm}]}\end{array}$ & $\begin{array}{c}\lambda_{I} \\
{[\mathrm{~cm}]}\end{array}$ & $\begin{array}{c}\mathrm{N} \\
{\left[\lambda_{I}\right]}\end{array}$ & $\begin{array}{c}10 \mathrm{GeV} \pi^{-} \\
\mathrm{rms} / \text { mean }\end{array}$ & $\begin{array}{c}20 \mathrm{GeV} \pi^{-} \\
\mathrm{rms} / \mathrm{mean}\end{array}$ & $\begin{array}{c}50 \mathrm{GeV} \pi^{-} \\
\mathrm{rms} / \text { mean }\end{array}$ & $\begin{array}{c}100 \mathrm{GeV} \pi^{-} \\
\mathrm{rms} / \text { mean }\end{array}$ & $\begin{array}{c}200 \mathrm{GeV} \pi^{-} \\
\mathrm{rms} / \mathrm{mean}\end{array}$ \\
\hline rms / mean & \multirow{2}{*}{7.13} & \multirow{2}{*}{1.12} & 21.88 & 5.8 & 0.155 & 0.163 & 0.172 & 0.185 & 0.192 \\
LCPhys BGO & & & & 5.8 & 0.101 & 0.110 & 0.120 & 0.137 & 0.157 \\
\hline LCPhys PbWO4 & 8.3 & & & 7 & 0.139 & 0.135 & 0.138 & 0.140 & 0.148 \\
& & & & 7 & 0.091 & 0.084 & 0.090 & 0.094 & 0.116 \\
\hline LCPhys d15 & 15 & & & & 0.109 & 0.086 & 0.064 & 0.056 & \\
& & & & & 0.074 & 0.051 & 0.029 & 0.035 & \\
\hline \hline
\end{tabular}

TABLE III: rms / mean (upper line) and Dual Readout corrected rms / mean (lower line) in BGO, PbWO4, d15 for $\pi^{-}$with 10, 20, 50, 100, or $200 \mathrm{GeV}$ energy. (see also Figure 6)
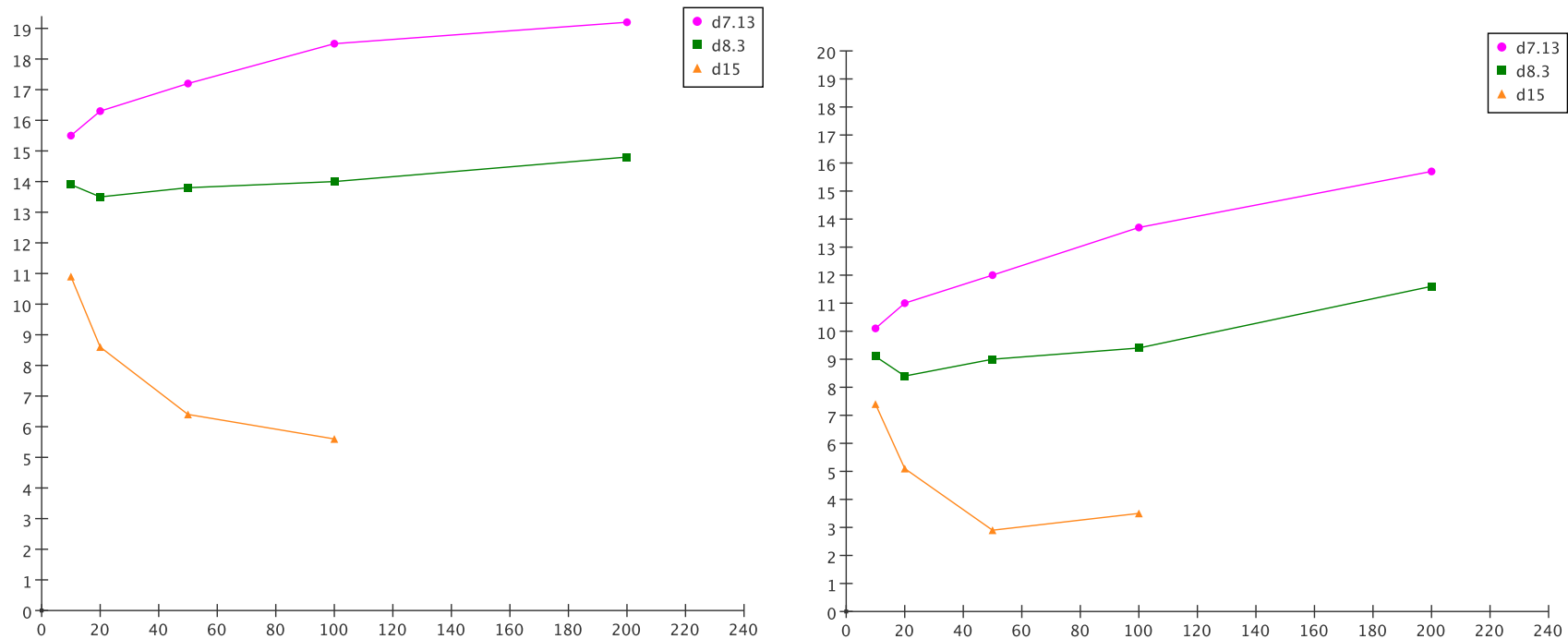

FIG. 6: rms/mean vs E (left) before and (right) after Dual Readout C/S Correction for crystal density $=7.13 \mathrm{BGO}, 8.3 \mathrm{PbWO} 4$, d15. (see also Table III) 


\begin{tabular}{|c|c|c|c|c|c|c|c|c|c|}
\hline Crystal & \begin{tabular}{|l|} 
Density \\
{$\left[\mathrm{g} / \mathrm{cm}^{3}\right]$} \\
\end{tabular} & $\begin{array}{c}\mathrm{X}_{0} \\
{[\mathrm{~cm}]}\end{array}$ & $\begin{array}{c}\lambda_{I} \\
{[\mathrm{~cm}]}\end{array}$ & $\begin{array}{c}\mathrm{N} \\
{\left[\lambda_{I}\right]} \\
\end{array}$ & $\begin{array}{l}10 \mathrm{GeV} \pi^{-} \\
\mathrm{rms} / \text { Edep }\end{array}$ & $\begin{array}{l}20 \mathrm{GeV} \pi^{-} \\
\mathrm{rms} / \text { Edep }\end{array}$ & $\begin{array}{l}50 \mathrm{GeV} \pi^{-} \\
\mathrm{rms} / \mathrm{Edep}\end{array}$ & $\begin{array}{l}100 \mathrm{GeV} \pi^{-} \\
\mathrm{rms} / \text { Edep }\end{array}$ & $\begin{array}{l}200 \mathrm{GeV} \pi^{-} \\
\mathrm{rms} / \mathrm{Edep}\end{array}$ \\
\hline $\mathrm{rms} /$ mean & & & & & & & & & \\
\hline LCPhys d7.13 & 7.13 & 1.12 & 21.88 & 5.8 & $1.09 / 7.77$ & $2.44 / 16.3$ & $6.73 / 41.1$ & $14.1 / 81.5$ & $29.9 / 161.5$ \\
\hline LCPhys $\mathrm{d} 8.3$ & 8.3 & & & 7 & $0.91 / 7.95$ & $1.89 / 16.7$ & $5.25 / 42.4$ & $11.4 / 84.5$ & $22.3 / 169.5$ \\
\hline LCPhys d10 & 10 & & & & $0.72 / 8.14$ & $1.44 / 17.1$ & $3.56 / 43.7$ & $7.60 / 87.6$ & $15.6 / 175.7$ \\
\hline LCPhys d 15 & 15 & & & & $0.63 / 8.40$ & $1.00 / 17.6$ & $1.78 / 45.2$ & $3.35 / 90.9$ & $6.57 / 183.0$ \\
\hline LCPhys d20 & 20 & & & & $0.62 / 8.52$ & $0.92 / 17.9$ & $1.57 / 45.7$ & $2.91 / 92.0$ & $4.86 / 185.2$ \\
\hline$\overline{E d e p ~(S) ~ / ~ E ~}$ & & & & & & & & & \\
\hline LCPhys d7.13 & 7.13 & 1.12 & 21.88 & 5.8 & 0.78 & 0.82 & 0.82 & 0.82 & 0.81 \\
\hline LCPhys $\mathrm{d} 8.3$ & 8.3 & & & 7 & 0.80 & 0.84 & 0.85 & 0.85 & 0.85 \\
\hline LCPhys d10 & 10 & & & & 0.81 & 0.86 & 0.87 & 0.88 & 0.88 \\
\hline LCPhys d 15 & 15 & & & & 0.84 & 0.88 & 0.90 & 0.91 & 0.92 \\
\hline LCPhys d20 & 20 & & & & 0.85 & 0.90 & 0.91 & 0.92 & 0.93 \\
\hline rms / mean & & & & & & & & & \\
\hline LCPhys d7.13 & 7.13 & 1.12 & 21.88 & 5.8 & 0.140 & 0.150 & 0.164 & 0.173 & 0.185 \\
\hline LCPhys d8.13 & 8.3 & & & 7 & 0.114 & 0.113 & 0.124 & 0.135 & 0.132 \\
\hline LCPhys d10 & 10 & & & & 0.088 & 0.084 & 0.081 & 0.087 & 0.089 \\
\hline LCPhys d 15 & 15 & & & & & 0.057 & 0.039 & 0.037 & 0.036 \\
\hline LCPhys d 20 & 20 & & & & 0.073 & 0.052 & 0.034 & 0.032 & 0.026 \\
\hline
\end{tabular}

TABLE IV: rms/mean of (Energy deposition, Edep (S)), and Edep(S) / E, in BGO, PbWO4, d15, d20 for $\pi^{-}$with $10,20,50,100$, or $200 \mathrm{GeV}$ energy. (Figure 7 )
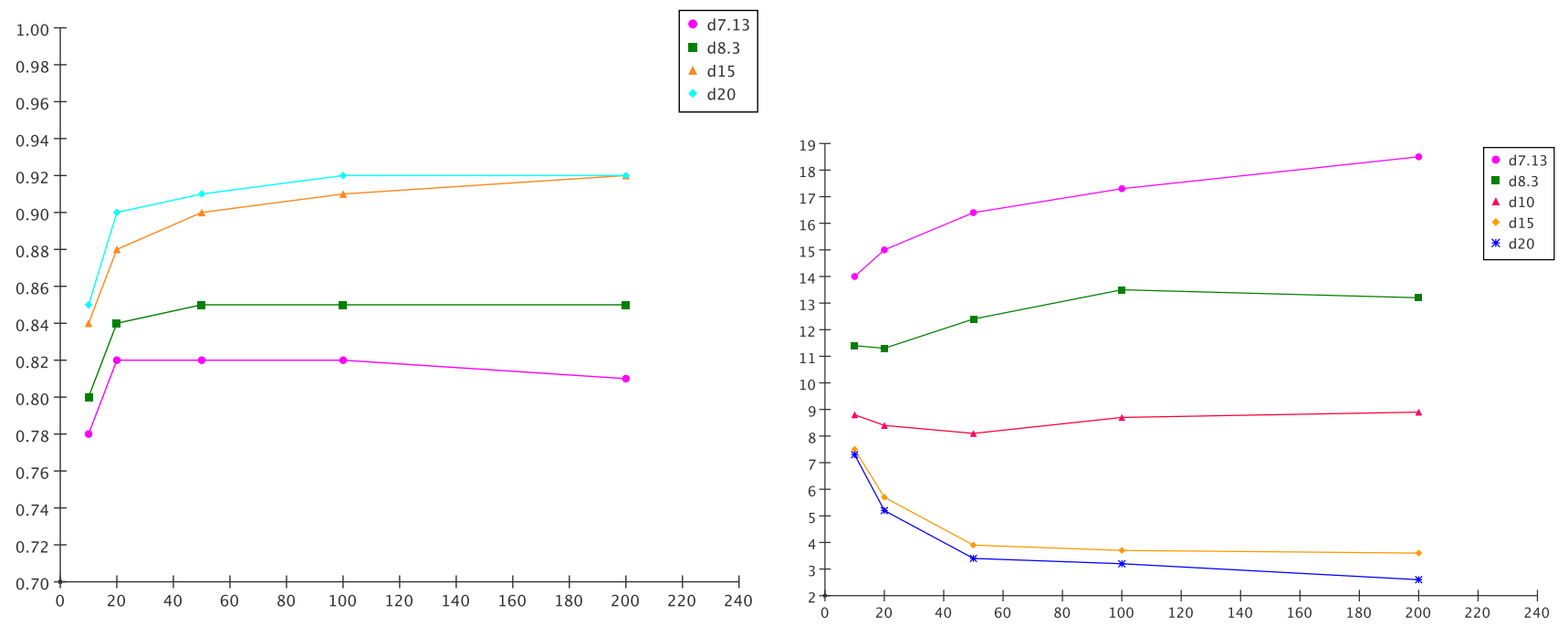

FIG. 7: S_Edep/E vs E, (left) and rms/mean vs E (right)

for crystal density $=7.13,8.3,15,20$ for $\pi^{-}$with $10,20,50,100,200 \mathrm{GeV}$. (Table IV) 


\section{B. LCPhys vs QGSP_BERT}

Some results using QGSP_BERT physics list in GEANT4 are shown in Table V. "LCPhys is a GEANT4 physics list written by Dennis Wright for simulation of events in ILC detectors." as described in http://silicondetector.org/display/ilc/What+is+LCPhys Other GEANT4 physics lists are shown in http://silicondetector.org/display/ilc/What+other+Geant4+physics+lists+does+SLIC+support

Comparing Table V and Table IV, some differences between QGSP_BERT and LCPhys and other parameter lists in GEANT4 are observed. For $10 \mathrm{GeV}$ pion, QGSP_BERT has energy deposition of $8.73 \mathrm{GeV}$ but LCPhys has 7.77 $\mathrm{GeV}$. Test Beam data will be needed to validate the GEANT4 physics lists and simulation.

\begin{tabular}{|c|c|c|c|c|c|c|c|c|c|}
\hline Crystal & $\begin{array}{l}\text { Density } \\
{\left[\mathrm{g} / \mathrm{cm}^{3}\right]}\end{array}$ & $\begin{array}{c}\mathrm{X}_{0} \\
{[\mathrm{~cm}]}\end{array}$ & $\begin{array}{c}\lambda_{I} \\
{[\mathrm{~cm}]}\end{array}$ & $\begin{array}{c}\mathrm{N} \\
{\left[\lambda_{I}\right]}\end{array}$ & $\begin{array}{l}10 \mathrm{GeV} \pi^{-} \\
\mathrm{rms} / \mathrm{Edep}\end{array}$ & $\begin{array}{l}20 \mathrm{GeV} \pi^{-} \\
\mathrm{rms} / \mathrm{Edep}\end{array}$ & $\begin{array}{l}50 \mathrm{GeV} \pi^{-} \\
\mathrm{rms} \mathrm{/} \mathrm{Edep}\end{array}$ & $\begin{array}{l}100 \mathrm{GeV} \pi^{-} \\
\mathrm{rms} / \mathrm{Edep}\end{array}$ & $\begin{array}{l}200 \mathrm{GeV} \pi^{-} \\
\mathrm{rms} / \mathrm{Edep}\end{array}$ \\
\hline $\begin{array}{ll}\text { QGSP_BERT } & \text { BGO } \\
\text { QGSP_BERT } & \text { d } 15\end{array}$ & $\begin{array}{l}7.13 \\
15\end{array}$ & & & 5.8 & $\begin{array}{c}1.16 / 8.73 \\
0.61 / 9.1\end{array}$ & $\begin{array}{l}2.53 / 17.9 \\
0.90 / 18.7\end{array}$ & $\begin{array}{l}6.99 / 44.4 \\
1.58 / 47.6\end{array}$ & $\begin{array}{l}15.5 / 86.8 \\
2.36 / 95.2\end{array}$ & \\
\hline $\begin{array}{ll}\text { Edep (S) / E } & \\
\text { QGSP_BERT } & \text { BGO } \\
\text { QGSP_BERT } & \text { d15 }\end{array}$ & $\begin{array}{l}7.13 \\
15\end{array}$ & & & 5.8 & $\begin{array}{l}0.87 \\
0.91\end{array}$ & $\begin{array}{l}0.89 \\
0.94\end{array}$ & $\begin{array}{l}0.89 \\
0.95\end{array}$ & $\begin{array}{l}0.87 \\
0.95\end{array}$ & \\
\hline $\begin{array}{ll}\mathrm{rms} / \mathrm{mean} & \\
\text { QGSP_BERT } & \text { BGO } \\
\text { QGSP_BERT } & \text { d } 15\end{array}$ & $\begin{array}{l}7.13 \\
15\end{array}$ & & & 5.8 & $\begin{array}{l}0.133 \\
0.067\end{array}$ & $\begin{array}{l}0.141 \\
0.048\end{array}$ & $\begin{array}{l}0.157 \\
0.033\end{array}$ & $\begin{array}{l}0.179 \\
0.025\end{array}$ & \\
\hline
\end{tabular}

TABLE V: rms/mean of Energy deposition, Edep (Figure 8) in BGO, d15 for $\pi^{-}$with 10, 20, 50, 100 GeV energy, using QGSP_BERT list in GEANT4
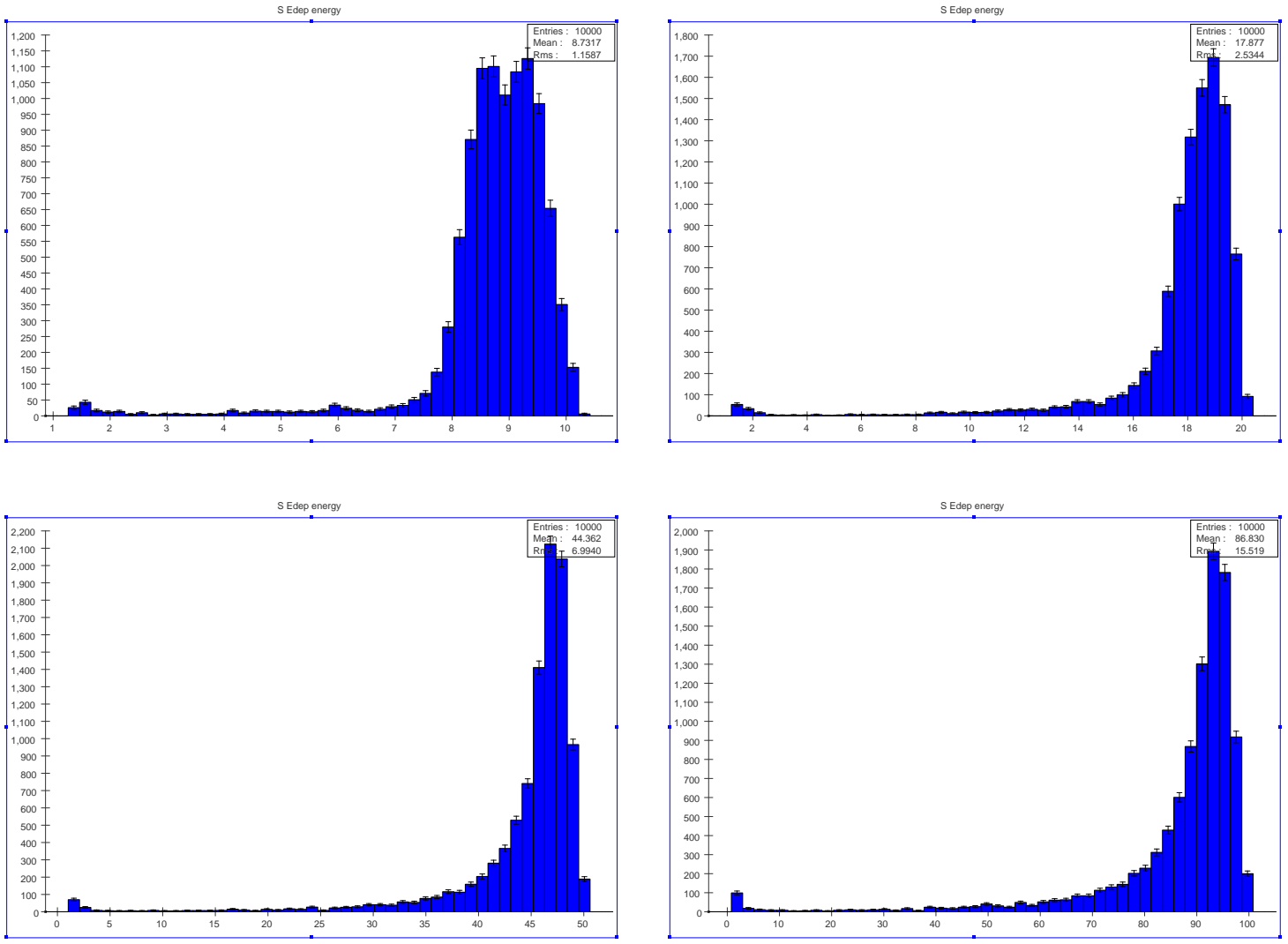

FIG. 8: 10, 20, $50100 \mathrm{GeV} \pi^{-}$energy deposition (Table V) in BGO, with QGSP_BERT 


\section{Segmentation}

An excellent example of PbWO4 crystal calorimeter is the CMS Electromagnetic calorimeter.[8] The density is $8.28 \mathrm{~g} / \mathrm{cm}^{3}$, radiation length $=0.89 \mathrm{~cm}$, Moliere radius $=2.2 \mathrm{~cm}$, Index of refraction $=2.2$. Peak emission $=440 \mathrm{~nm}$, $85 \%$ of Light emission is within 25nsec, and Light Yield is 100 photons/MeV. About 4.5 photoelectrons are collected per $\mathrm{MeV}$ in both the barrel and the endcap. Each Central and/or Barrel crystal is $23 \mathrm{~cm}$ long corresponding to 25.8 $\mathrm{X}_{0}$ (radiation lengths), with front size $=2.2 \times 2.2 \mathrm{~cm}^{2}$ and back size $=2.6 \times 2.6 \mathrm{~cm}^{2}$. The total crystal volume is $8.14 \mathrm{~m}^{3}$, and the total mass is 67.4 tons. A dual readout PbWO4 crystal hadron or total absorption calorimeter would be geq 10 times the size of the CMS PbWO4 EM calorimeter.

The L3 EM calorimeter[7] BGO crystals had front size $=2 \times 2 \mathrm{~cm}^{2}$, back size $=3 \times 3 \mathrm{~cm}^{2}, 24 \mathrm{~cm}$ length, similar area and length as the CMS EM calorimeter PbWO4 crystals.

Hadron showers are contained transversely within a radius of one interaction length from the trajectory of the hadron. Hadron showers have large longitudinal fluctuations over $\geq 8$ interaction lengths, and large fluctuations at the end of the shower. Longitudinal segmentation of the dual readout crystal hadron calorimeter should be studied with more realistic simulation including light yields, photon detection efficiency, and support for the 700 tons of crystals.

Because of this study, Adam Para and Hans Wenzel wrote a script to enable creating crystal detectors with different crystal density and/or different transverse segmentation. The significant effects of crystal density have been presented in this note.

Transverse segmentation is not expected to have significant effects on hadron energy resolution, especially for the present CCAL02 simulation which simulates $\sim 100 \%$ photon detection efficiency. Transverse segmentation has some effects on jet mass resolution, which is being studied using $W \rightarrow$ jets events or $Z \rightarrow j e t s$ events. Studies with more realistic simulation and longitudinal segmentation will be much more important.

In this short study, the EM calorimeter crystals are $1.5 \times 1.5 \mathrm{~cm}^{2}, 3 \times 3 \mathrm{~cm}^{2}, 4.5 \times 4.5 \mathrm{~cm}^{2}, \mathrm{HA} 2.5 \times 2.5 \mathrm{~cm}^{2}$, $5 \times 5 \mathrm{~cm}^{2}, 7.5 \times 7.5 \mathrm{~cm}^{2}$. The shape of the crystals should be truncated pyramid.

\begin{tabular}{l|c|c|c|c|c|c|c|c|c}
\hline \hline Crystal & $\begin{array}{c}\text { Density } \\
{\left[\mathrm{g} / \mathrm{cm}^{3}\right]}\end{array}$ & $\begin{array}{c}\mathrm{X}_{0} \\
{[\mathrm{~cm}]}\end{array}$ & $\begin{array}{c}\lambda_{I} \\
{[\mathrm{~cm}]}\end{array}$ & $\begin{array}{c}\mathrm{N} \\
{\left[\lambda_{I}\right]}\end{array}$ & $\begin{array}{c}10 \mathrm{GeV} \pi^{-} \\
\mathrm{rms} / \text { Edep }\end{array}$ & $\begin{array}{c}20 \mathrm{GeV} \pi^{-} \\
\mathrm{rms} / \mathrm{Edep}\end{array}$ & $\begin{array}{c}50 \mathrm{GeV} \pi^{-} \\
\mathrm{rms} / \mathrm{Edep}\end{array}$ & $\begin{array}{l}100 \mathrm{GeV} \pi^{-} \\
\mathrm{rms} / \text { Edep }\end{array}$ & $\begin{array}{l}200 \mathrm{GeV} \pi^{-} \\
\mathrm{rms} / \text { Edep }\end{array}$ \\
LCPhys d7_25_15 & 7.13 & & & 5.8 & $7.8 \pm 0.01$ & & $41.1 \pm 0.07$ & $81.3 \pm 0.14$ & \\
LCPhys d7_50_30 & 7.13 & 1.12 & 21.88 & 5.8 & $1.09 / 7.77$ & $2.44 / 16.3$ & $6.73 / 41.1$ & $14.1 / 81.5$ & \\
LCPhys d7_75_45 & 7.13 & & & 5.8 & $1.09 / 7.77$ & $2.45 / 16.3$ & $6.68 / 41.0$ & $15.2 / 81.6$ & \\
\hline LCPhys d15_25_15 & 15 & & & & $0.63 / 8.40$ & $1.01 / 17.6$ & $1.80 / 45.1$ & $3.36 / 90.9$ & \\
LCPhys d15_50_30 & 15 & & & & $7.5 \pm 0.01$ & $16.2 \pm 0.01$ & $42.1 \pm 0.03$ & $85.5 \pm 0.05$ & \\
LCPhys d15_75_45 & 15 & & & & $0.63 / 8.40$ & $1.00 / 17.6$ & $1.81 / 45.1$ & $3.29 / 90.9$ & \\
LCPhys d20_25_15 & 20 & & & & $8.5 \pm 0.01$ & $17.9 \pm 0.01$ & $45.7 \pm 0.02$ & $92.0 \pm 0.03$ & \\
\hline \hline
\end{tabular}

TABLE VI: rms/mean of Energy deposition, Edep, in BGO, PbWO4, d15 for $\pi^{-}$with 10, 20, 50, 100, or $200 \mathrm{GeV}$ energy, for 3 transverse sizes of crystals.

The effect of magnetic field to jet and di-jet mass reconstruction has been studied[10] using single W Monte Carlo events, with $\mathrm{W}$ momentum uniformly distributed between 0 and $100 \mathrm{GeV} / \mathrm{c}$. Strong magnetific field induces a sizable contribution to di-jet mass resolution. The bending of charged particles in a $5 \mathrm{~T}$ field affects the mass resolution larger than the the effect of the dual readout calorimeter energy resolution. The change of the invariant mass of a system in a magnetic field is calculable for each individual event using track trajectory and momentum, and the location and energy deposition at the impact point at the calorimeter, The correction for magnetic field is simple. In this short study, we do not repeat the magnetic field tracking correction study, we just show some results before and after the tracking correction. Much more detailed studies of jet and di-jet mass resolution should be done with more realistic simulation of the detector.

Dual Readout reconstructed $\mathrm{Z}$ mass (without jet clustering), for 5,000 $Z \rightarrow$ jets before and after tracking correction for B field are shown in Figure 9 for $Z$ bosons with $P_{t}=50,100 \mathrm{GeV} / \mathrm{c}$ and in Figure 10 for $Z$ bosons with $P_{t}=200 \mathrm{GeV} / \mathrm{c}$. The over-correction in Figure 10 is also observed in Figure 20. More work is needed for crystal dual readout correction, for type(density) of crystal, GEANT4 physics list, and energy dependence. Dual Readout reconstructed $\mathrm{W}$ and $\mathrm{Z}$ mass (without jet clustering), for 5,000 W $\rightarrow$ jets and 5,000 $\mathrm{Z} \rightarrow$ jets are shown in FIG. 11. 

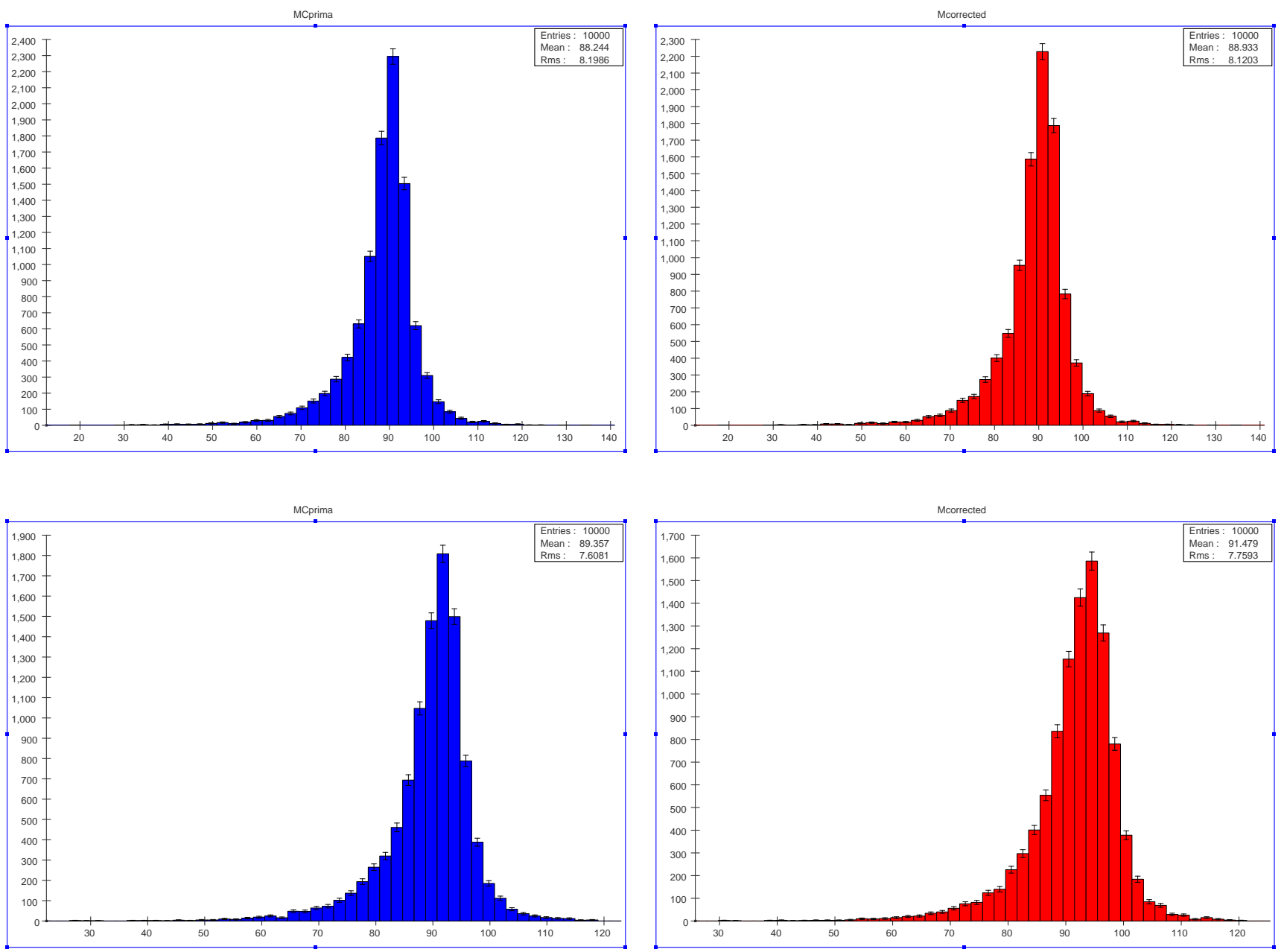

FIG. 9: BGO dual readout corrected $\mathrm{Z}$ mass, before (left) and after (right) tracking correction. Top: $\mathrm{Z}$ with $P_{t}=50 \mathrm{GeV} / \mathrm{c}$ Bottom: $\mathrm{Z}$ with $P_{t}=100 \mathrm{GeV} / \mathrm{c}$
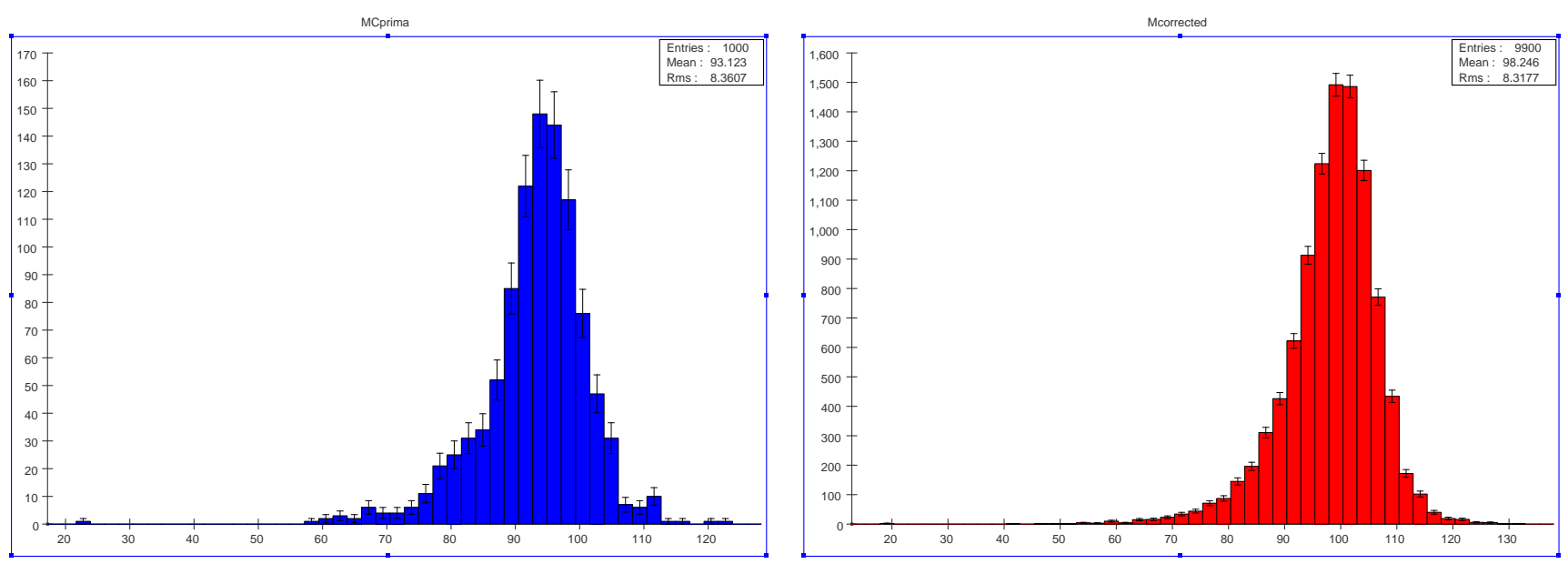

FIG. 10: BGO dual readout corrected $\mathrm{Z}$ mass, before and after tracking correction, for $\mathrm{Z}$ with $P_{t}=200 \mathrm{GeV} / \mathrm{c}$. 


\begin{tabular}{|c|c|c|c|c|c|}
\hline Jets & $\overline{\mathrm{P}}$ & Mass & $\mathrm{rms}$ & M_corrected & $\mathrm{rms}$ \\
\hline W_0_100 & $0-100$ & 77.68 & 6.30 & 79.54 & 6.72 \\
\hline W_100_200 & $100-200$ & 80.85 & 6.89 & 86.72 & 8.31 \\
\hline Z_0_100 & $0-100$ & 86.58 & 8.64 & 88.21 & 8.86 \\
\hline Z_10-250 & $10-250$ & 77. & 6. & 79. & 6. \\
\hline $\mathrm{Z} 30 \times 30,50 \times 50$ & 50 & 88.24 & 8.19 & 88.93 & 8.12 \\
\hline Z 30x30, 50x50 & 100 & 89.36 & 7.61 & 91.45 & 7.76 \\
\hline Z $30 \times 30,50 \times 50$ & 200 & 93.12 & 8.36 & 98.25 & 8.32 \\
\hline $\mathrm{Z} 15 \times 15,25 \times 25$ & 50 & 85.76 & 14.37 & 86.44 & 14.21 \\
\hline 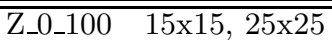 & $\overline{0-100}$ & 84.97 & 12.79 & 86.61 & 12.96 \\
\hline Z_0_100 30x30, 50x50 & $0-100$ & 78.65 & 11.83 & 80.23 . & 11.99 \\
\hline Z_0_100 $45 \times 45,75 \times 75$ & $0-100$ & 78.56 & 11.84 & 80.19 & 12.01 \\
\hline$\overline{\text { W_0_100 }} 15 \times 15,25 \times 25$ & $0-100$ & 76.44 & 10.42 & 78.30 & 10.80 \\
\hline W_0_100 30x30, 50x50 & $0-100$ & 70.90 & 9.47 & 72.77 & 9.82 \\
\hline W_0_100 $45 \times 45,75 \times 75$ & $0-100$ & 70.81 & 9.59 & 72.68 & 9.95 \\
\hline
\end{tabular}

TABLE VII: W or Z mass from Dual Readout BGO Calorimeter CCAL02, from GEANT4 QGSP_BERT, before and after tracking correction for 5 Tesla B-field.

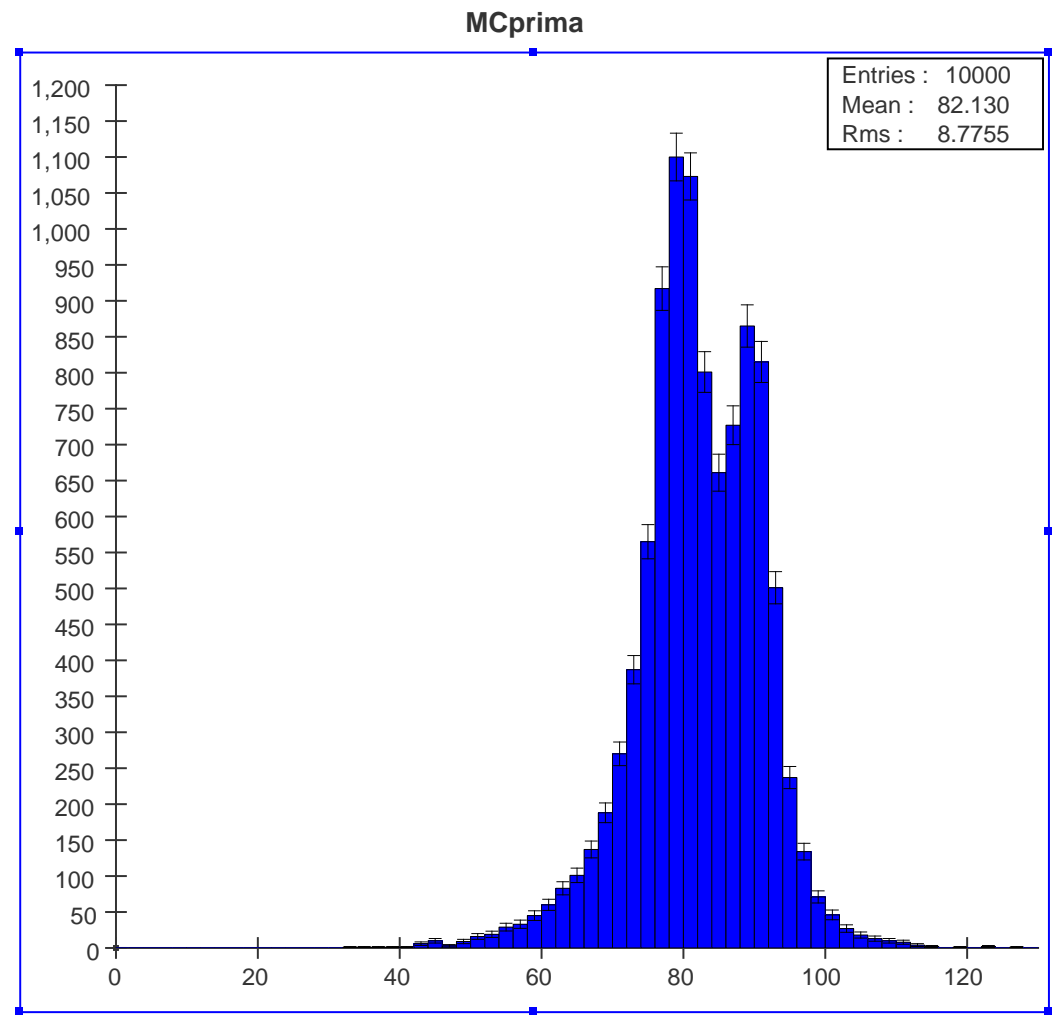

FIG. 11: Dual Readout reconstructed $\mathrm{W}$ and $\mathrm{Z}$ mass (without jet clustering), for $5,000 \mathrm{~W} \rightarrow$ jets and $5,000 Z \rightarrow$ jets.

The relative number of $W$ and/or $Z$ events will depend on the physics processes at the future lepton collider. It is important to achieve the goal of $30 \% / \sqrt{E}$ jet energy resolution.

Longitudinal segmentation of the dual readout crystal hadron calorimeter should be studied with more realistic simulation including light yields, photon detection efficiency, and support for the 700 tons of crystals. 


\section{SUMMARY}

Studies of requirements and specifications of crystals are necessary to develop a new generation of crystals for dual readout crystal hadron or total absorption calorimeter. This has been a short and basic comparison study of PbWO4 and BGO as candidates for Dual Readout Crystal hadron calorimeter. The cost of $\mathrm{PbWO} 4$ is less prohibitive than BGO. A calorimeter with PbWO4 would have more interaction lengths, for more hadron shower containment than with BGO. Hadron shower energy leakage is clearly observed for BGO with 5.8 interaction lengths and PbWO4 with $7 \lambda_{I}$, but not for d15 and d20 crystals with densities of $15 \mathrm{~g} / \mathrm{cm}^{3}$ or $20 \mathrm{~g} / \mathrm{cm}^{3}$. With 5.8 or 7 interaction lengths, the hadron shower leakage significantly degrades the hadron energy resolution before and/or after dual readout correction. Calorimeters at the Muon Collider would require at least 8 interaction lengths.

The detector simulation should have more realistic geometry than the present CCAL02. The simulation should include boundaries between crystals including for the support structure for the 700 tons of crystals, and should simulate the photo-detectors and associated efficiencies. The simulation should enable varying longitudinal segmentation for the important longitudinal segmentation and calibration studies. More complete simulation with crystal Light Yield, Decay Time, digitization and optical readout efficiencies and noise will be needed for calibration and energy resolution studies of the feasibility of dual readout crystal total absorption or hadron calorimeter.

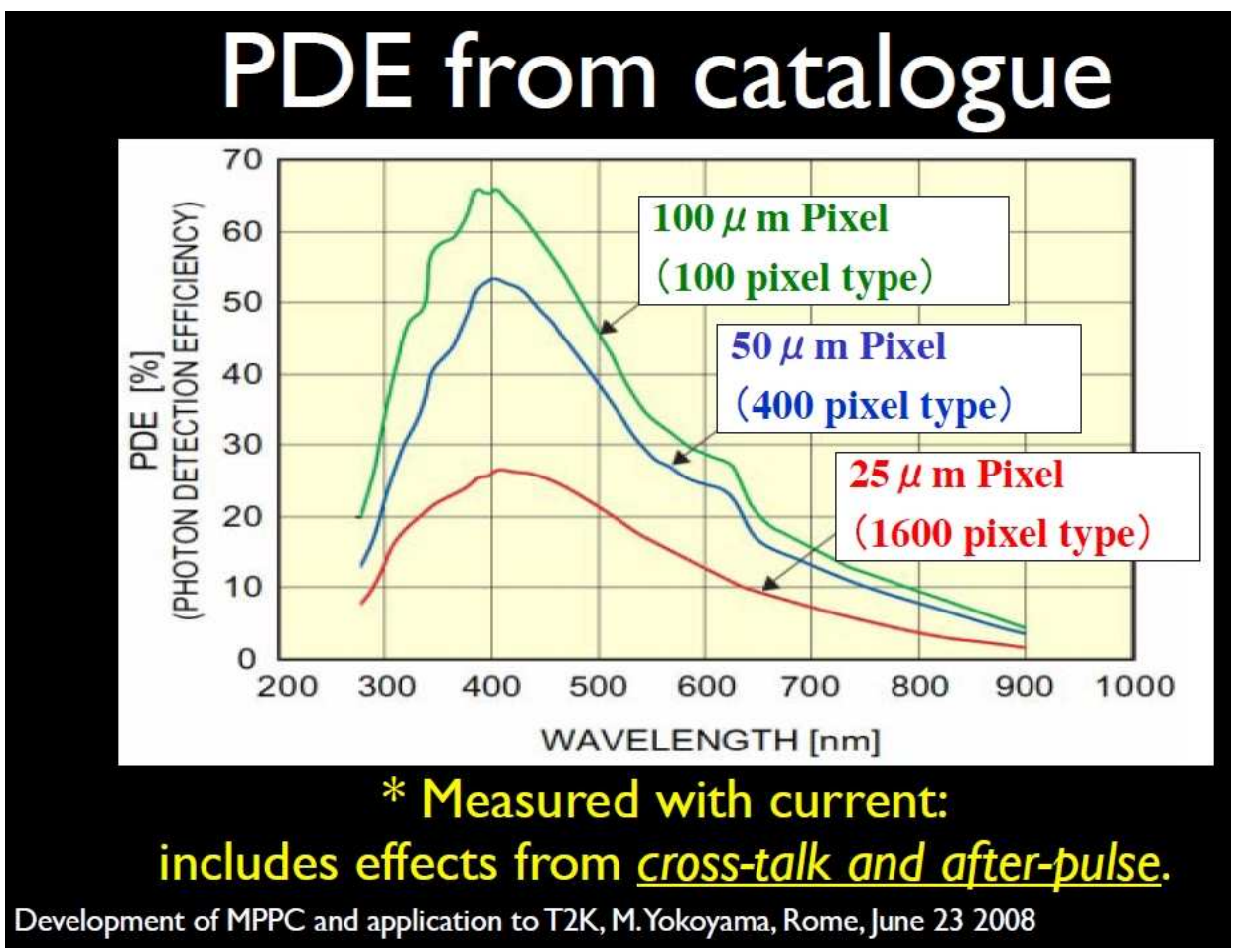

FIG. 12: Photon detection efficiency of MPPC/SiPM, including cross-talk and after-pulse (20-30\% over-estimate).[16]

Light yields of crystals, Photon detection efficiency, longitudinal segmentation, crystal support structure, and more realistic geometry should be implemented in the simulation. 


\section{ACKNOWLEDGMENTS}

Thanks to Hans Wenzel and Adam Para for their important help with the dual readout crystal calorimeter simulation and analysis software for this study.

\section{REFERENCES}

[1] All dual-readout work by the DREAM collaboration has been led by Dr. R. Wigmans, and all of the results of this work are published in Nucl. Instrs. Meths., Akchurin, et al.

(a) "Hadron and Jet Detection with a Dual-Readout Calorimeter", NIM A537 (2005) 537-561.

(b) "Electron Detection with a Dual-Readout Calorimeter", NIM A536 (2005) 29-51.

(c) “Muon Detection with a Dual-Readout Calorimeter", NIM A533 (2004) 305-321.

(d) "Comparison of High-Energy Electromagnetic Shower Profiles Measured with Scintillation and Cerenkov Light", NIM A548 (2005) 336-354.

(e) "Separation of Scintillation and Cerenkov Light in an Optical Calorimeter", NIM A550 (2005) 185-200.

(f) "Measurement of the Contribution of Neutrons to Hadron Calorimeter Signals," NIM A581 (2007) 643.

(g) "Contributions of CerenkovLight to the Signals from Lead Tungstate Crystals," NIM A582 (2007) 474.

(h) "Comparison of High-Energy Hadronic Shower Profiles Measured with Scintillation and Cerenkov Light", NIM A584 (2008) 304.

(i) "Dual-Readout Calorimetry with Lead Tungstate Crystals", NIM A584 (2008) 273.

(j) "Effects of the Termperature Dependence of the Signals from Lead Tungstate Crystals", NIM A593 (2008) 530.

(k) "Separation of Crystal Signals into Scintillation and CerenkovComponents", NIM A595 (2008) 359.

(1) "Neutron Signals for Dual-Readout Calorimetry", NIM A598(2009) 422.

(m) "Dual-Readout Calorimetry with Crystal Calorimeters", NIM A598(2009) 710.

(n) "New Crystals for Dual-Readout Calorimetry," NIM A604 (2009) 512.

(o) "Dual-Readout Calorimetry with a Full-Size Electromagnetic Section," submitted to NIM.

[2] 4th Concept Detector, http://www.4thconcept.org/4LoI.pdf http://www.4thconcept.org/4IDAG.pdf

[3] Muon Collider Physics Workshop, http://indico.fnal.gov/conferenceDisplay.py?confId=2855

[4] Dual Readout calorimeter for the Muon Collider

John Hauptman, "Detector Design Strategies",

http://indico.fnal.gov/materialDisplay.py?contribId=67\&sessionId=22\&materialId=slides\&confId $=2855$

Corrado Gatto, "Simulation and Performance of Detectors",

http://indico.fnal.gov/materialDisplay.py?contribId=37\&sessionId=12\&materialId=slides\&confId=2855

Corrado Gatto, ILCroot: "Infrastructure for Large Collider based on root",

http://indico.fnal.gov/materialDisplay.py?contribId=74\&sessionId=13\&materialId $=$ slides\&confId $=2855$

Anna Mazzacane, "W-Z Reconstruction",

http://indico.fnal.gov/materialDisplay.py?contribId=38\&sessionId=13\&materialId=slides\&confId = 2855

John Hauptman, "Dual Readout Calorimetry",

http://indico.fnal.gov/materialDisplay.py?contribId=31\&sessionId=17\&materialId=slides\&confId $=2855$

Vito Di Benedetto, "Dual Readout Calorimetry in ILCROOT", 
http://indico.fnal.gov/materialDisplay.py?contribId=32\&sessionId=17\&materialId =slides\&confId $=2855$ and many presentations from 4th Concept Detector and DREAM since 2005.

[5] Hans Wenzel, Crystal Calorimetry http://indico.fnal.gov/materialDisplay.py?contribId=33\&sessionId=17\&materialId=slides\&confId $=2855$

[6] ALCPG09, http://panda.unm.edu/LCWA09/ http://ilcagenda.linearcollider.org/conferenceDisplay.py?confId=3461

[7] The L3 Electromagnetic Calorimeter, http://wwwlapp.in2p3.fr/preplapp/psexp/lappexp9502.ps.gz

[8] CMS Collaboration, http://cmsdoc.cern.ch/cms/Publications/detpaper/CMS-JINST.pdf

[9] Hans Wenzel, "Implementing a dual readout calorimeter in SLIC and testing Geant4 Physics", http://ilcagenda.linearcollider.org/materialDisplay.py?contribId=77\&sessionId $=11 \&$ materialId $=$ slides\&confId $=3461$

[10] Adam Para, "Jets and Jet-Jet Mass Reconstruction in a Crystal Calorimeter", http://ilcagenda.linearcollider.org/materialDisplay.py?contribId=324\&sessionId=6\&materialId $=$ slides $\&$ confId $=3461$

[11] Ren-Yuan Zhu, "Crystal Calorimeters in the next decade", http://www.hep.caltech.edu/ zhu/talks/ryz_091029_N43-1.pdf

[12] Alexander Gektin, "New generation of HEP scintillator: search and development criteria", http://www-ppd.fnal.gov/eppoffice-w/Research_Techniques_Seminar/ Oct. 27, 2009

[13] A quotation from Saint Gobain for large quantity purchase of BGO

[14] Dieter Schlatter, "Detector Concept for CLIC", http://ilcagenda.linearcollider.org/materialDisplay.py?contribId=39\&sessionId=4\&materialId=slides\&confId=3154

[15] Fermilab E594, http://lss.fnal.gov/archive/1987/pub/Pub-87-159-E.pdf

[16] Yokoyama, M., Geiger-mode APD, SiPM, MPPC, http://agenda.infn.it/getFile.py/access?sessionId $=1 \&$ resId $=0 \&$ materialId $=0 \&$ confId $=1530$ http://agenda.infn.it/getFile.py/access?resId=0\&materialId=slides\&confId =539 


\section{APPENDIX A: ADDITIONAL PLOTS}

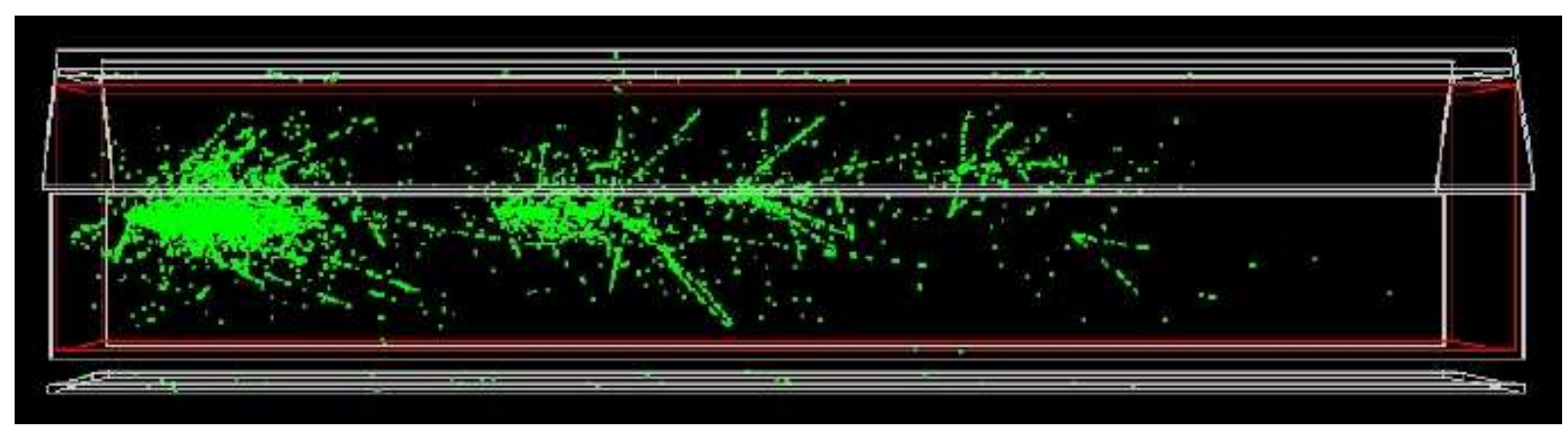

FIG. 13: Simulation of $100 \mathrm{GeV} \pi^{-}$shower in the DREAM daul readout Calorimeter, which has 10 interaction lengths[4]

How many interaction length for HCAL?

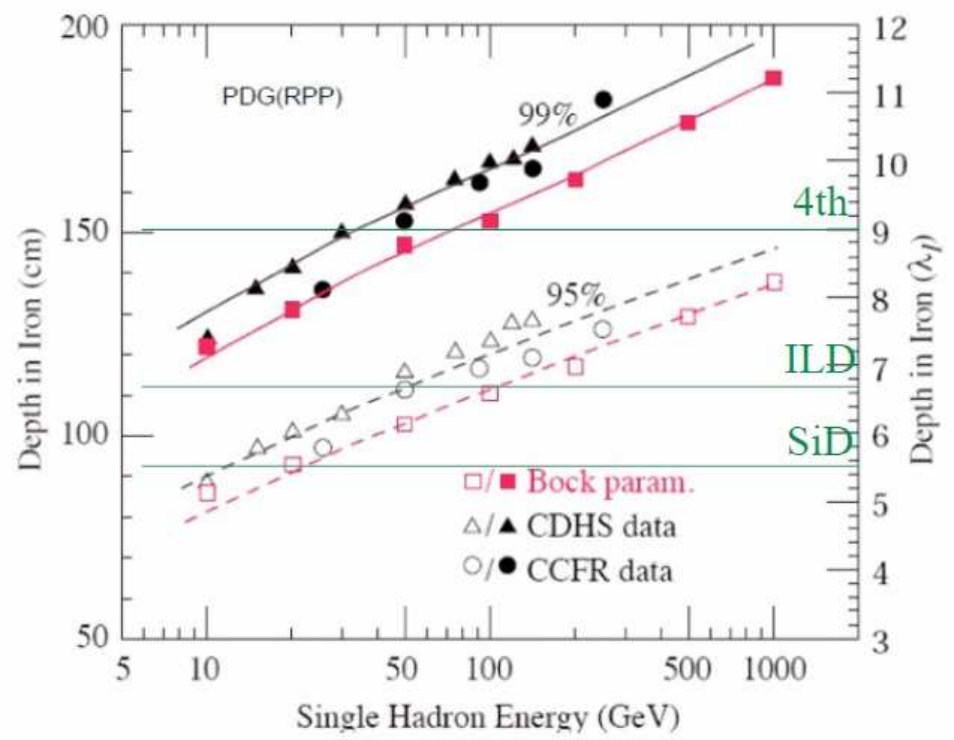

\begin{tabular}{|c|c|c|}
\hline ILD & SiD & 4 th \\
\hline $5.7 \lambda$ & $4.5 \lambda$ & $8.0 \lambda$ \\
\hline ECAL $~$ & $1 \lambda$ & $1.3 \lambda$ \\
\hline
\end{tabular}

Expl: to absorb $95 \%$ of the energy of a $300 \mathrm{GeV}$ pion $\rightarrow 8 \lambda_{\mathrm{I}}$

\begin{tabular}{|c|c|c|c|}
\hline & W & U & Fe \\
\hline $\begin{array}{c}\text { density } \\
{\left[\mathrm{g} / \mathrm{cm}^{2}\right]}\end{array}$ & 19.3 & 19.0 & 7.9 \\
\hline$\lambda_{\text {Int }}[\mathrm{cm}]$ & 10.0 & 11.0 & 16.8 \\
\hline $\mathrm{X}_{0}[\mathrm{~cm}]$ & 0.3 & 0.3 & 1.8 \\
\hline
\end{tabular}

FIG. 14: Interaction Lengths for Hadron calorimeter[14] 


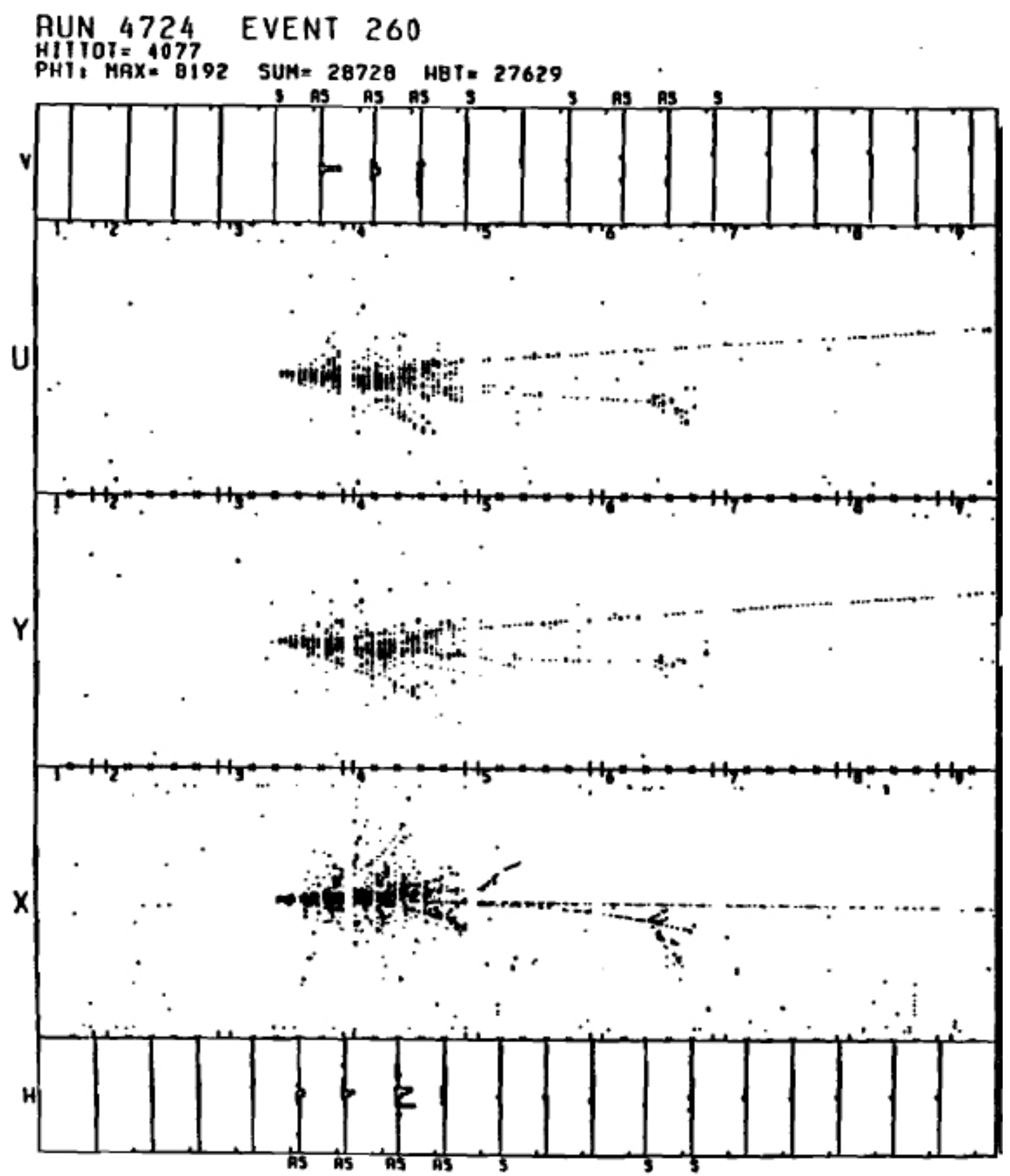

FIG. 15: Typical Hadron shower in E594 neutrino interaction.[15] The calorimeter had 22 interaction lengths. 


\begin{tabular}{|c|c|c|c|c|c|c|c|c|}
\hline Crystal & $\operatorname{Nal}(\mathrm{TI})$ & CsI(TI) & Csl & $\mathrm{BaF}_{2}$ & BGO & LYSO(Ce) & PWO & $\mathrm{PbF}_{2}$ \\
\hline Density $\left(\mathrm{g} / \mathrm{cm}^{3}\right)$ & 3.67 & 4.51 & 4.51 & 4.89 & 7.13 & 7.40 & 8.3 & 7.77 \\
\hline Melting Point $\left({ }^{\circ} \mathrm{C}\right)$ & 651 & 621 & 621 & 1280 & 1050 & 2050 & 1123 & 824 \\
\hline Radiation Length (cm) & 2.59 & 1.86 & 1.86 & 2.03 & 1.12 & 1.14 & 0.89 & 0.93 \\
\hline Molière Radius (cm) & 4.13 & 3.57 & 3.57 & 3.10 & 2.23 & 2.07 & 2.00 & 2.21 \\
\hline Interaction Length $(\mathrm{cm})$ & 42.9 & 39.3 & 39.3 & 30.7 & 22.8 & 20.9 & 20.7 & 21.0 \\
\hline Refractive Index a & 1.85 & 1.79 & 1.95 & 1.50 & 2.15 & 1.82 & 2.20 & 1.82 \\
\hline Hygroscopicity & Yes & Slight & Slight & No & No & No & No & No \\
\hline $\begin{array}{l}\text { Luminescence }^{b}(\mathrm{~nm}) \text { (at } \\
\text { peak) }\end{array}$ & 410 & 550 & $\begin{array}{l}420 \\
310\end{array}$ & $\begin{array}{l}300 \\
220\end{array}$ & 480 & 402 & $\begin{array}{l}425 \\
420\end{array}$ & $?$ \\
\hline Decay Time ${ }^{b}$ (ns) & 245 & 1220 & $\begin{array}{c}30 \\
6\end{array}$ & $\begin{array}{l}650 \\
0.9\end{array}$ & 300 & 40 & $\begin{array}{l}30 \\
10\end{array}$ & $?$ \\
\hline Light Yield b,c (\%) & 100 & 165 & $\begin{array}{l}3.6 \\
1.1\end{array}$ & $\begin{array}{l}36 \\
4.1\end{array}$ & 21 & 85 & $\begin{array}{l}0.3 \\
0.1\end{array}$ & $?$ \\
\hline $\mathrm{d}(\mathrm{LY}) / \mathrm{dT} \mathrm{T}^{\mathrm{b}}\left(\% /{ }^{\circ} \mathrm{C}\right)$ & -0.2 & 0.4 & -1.4 & $\begin{array}{c}-1.9 \\
0.1\end{array}$ & -0.9 & -0.2 & -2.5 & $?$ \\
\hline Experiment & $\begin{array}{c}\text { Crystal } \\
\text { Ball }\end{array}$ & $\begin{array}{l}\text { BaBar } \\
\text { BELLE } \\
\text { BES III }\end{array}$ & $\mathrm{KTeV}$ & $\begin{array}{l}\left(L^{*}\right) \\
\text { (GEM) } \\
\text { TAPS }\end{array}$ & $\begin{array}{l}\text { L3 } \\
\text { BELLE }\end{array}$ & $\begin{array}{l}\text { KLOE-2 } \\
\text { SuperB } \\
\text { SLHC? }\end{array}$ & $\begin{array}{c}\text { CMS } \\
\text { ALICE } \\
\text { PANDA }\end{array}$ & HHCAL? \\
\hline \multicolumn{9}{|c|}{ a. at peak of emission; b. up/low row: slow/fast component; c. QE of readout device taken out. } \\
\hline
\end{tabular}

FIG. 16: crystals for calorimeter[11] 

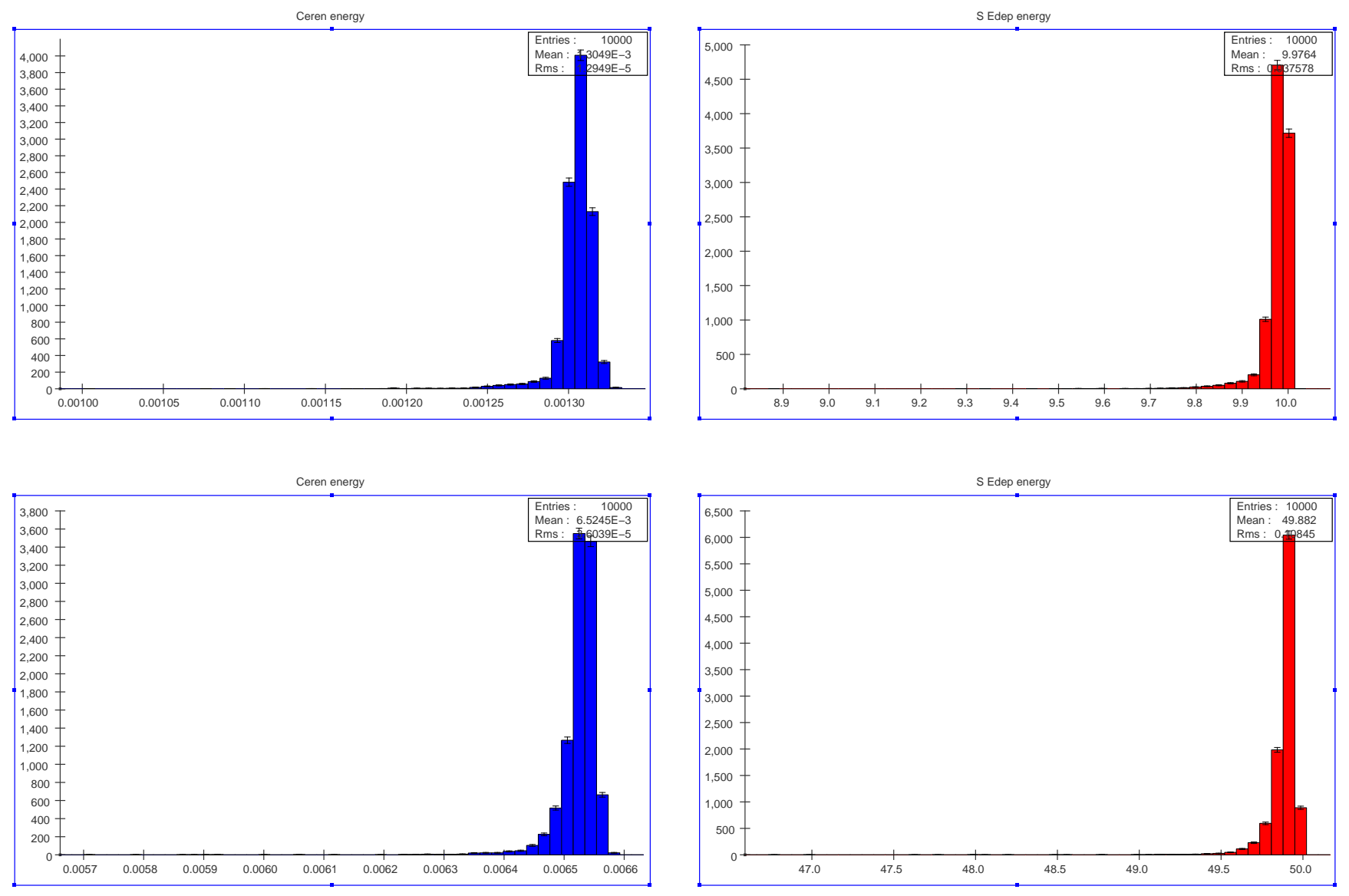

FIG. 17: LCPhys 25_15 Cerenkov (left) and Scintillation (right) energy deposition. Top: $10 \mathrm{GeV} e^{-}$; Bottom: $50 \mathrm{GeV} e^{-}$ 
IX. APPENDIX B: RESULTS FROM HANS WENZEL

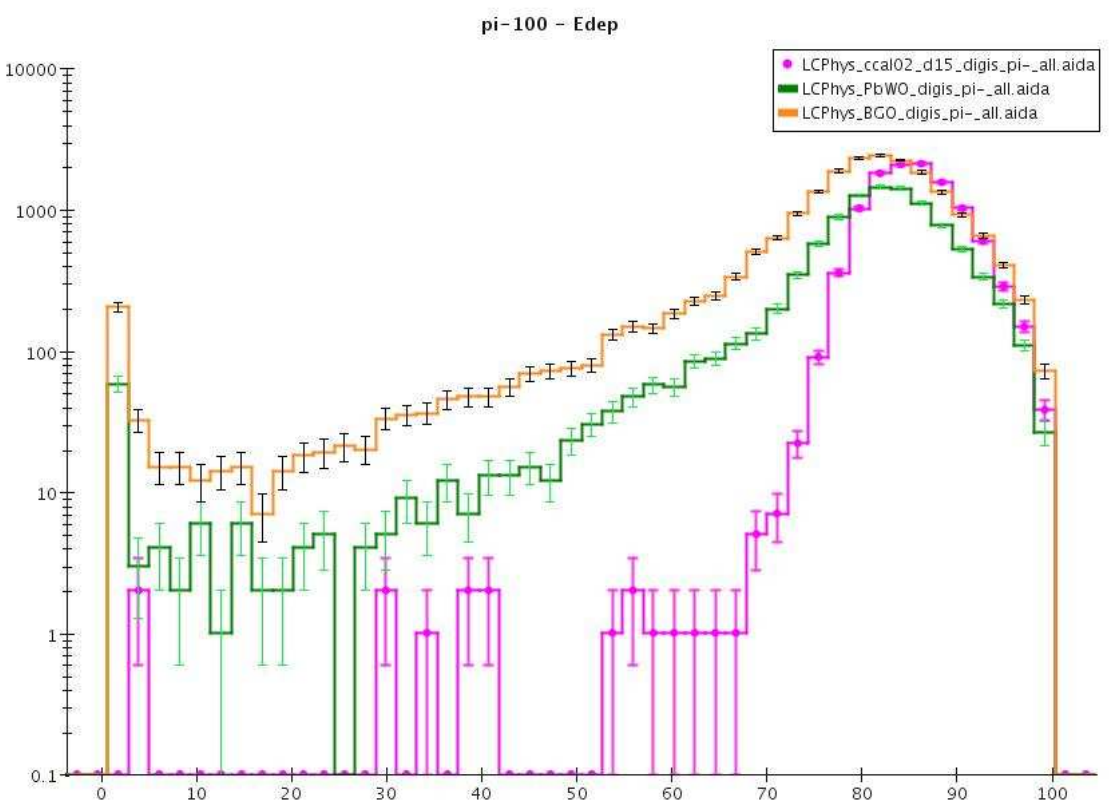

FIG. 18: Leakage also observed by Hans Wenzel

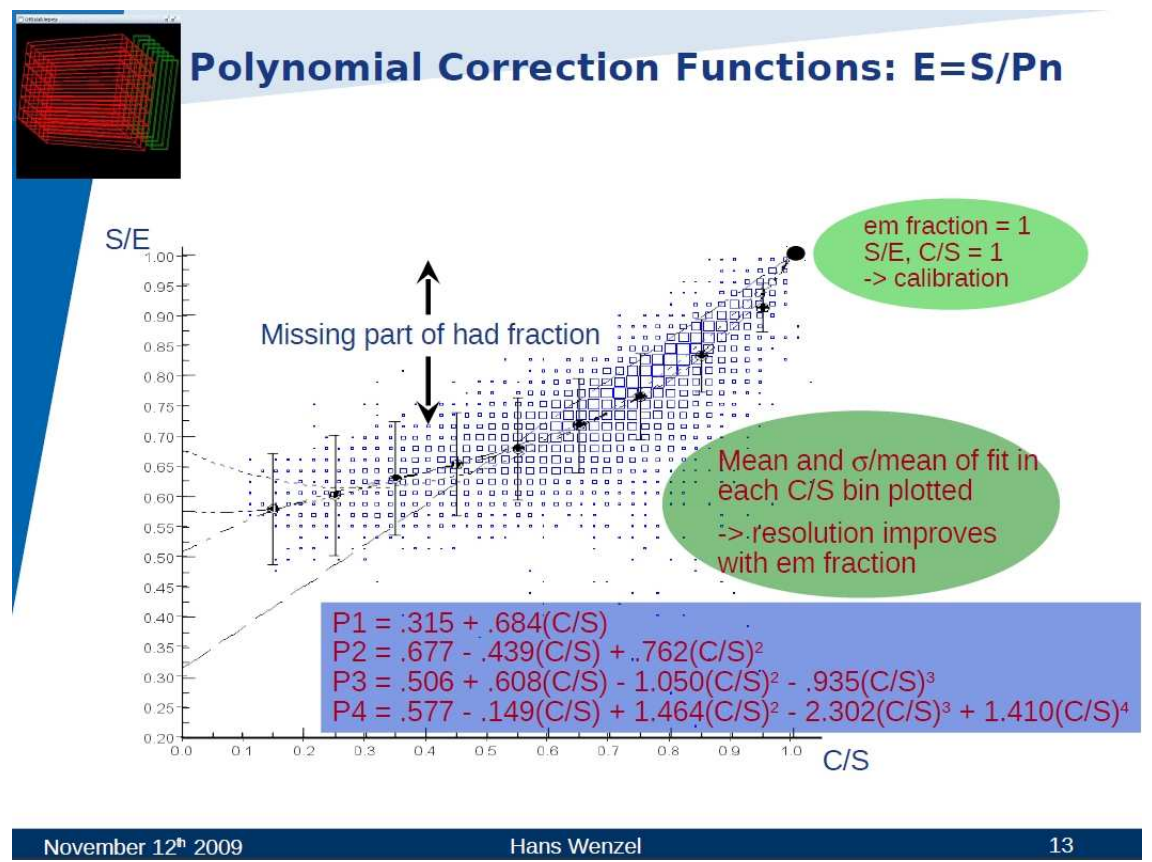

FIG. 19: Leakage also observed by Hans Wenzel 
LCPhys_ccal02_d15_digis_pi-_all_resol.aida

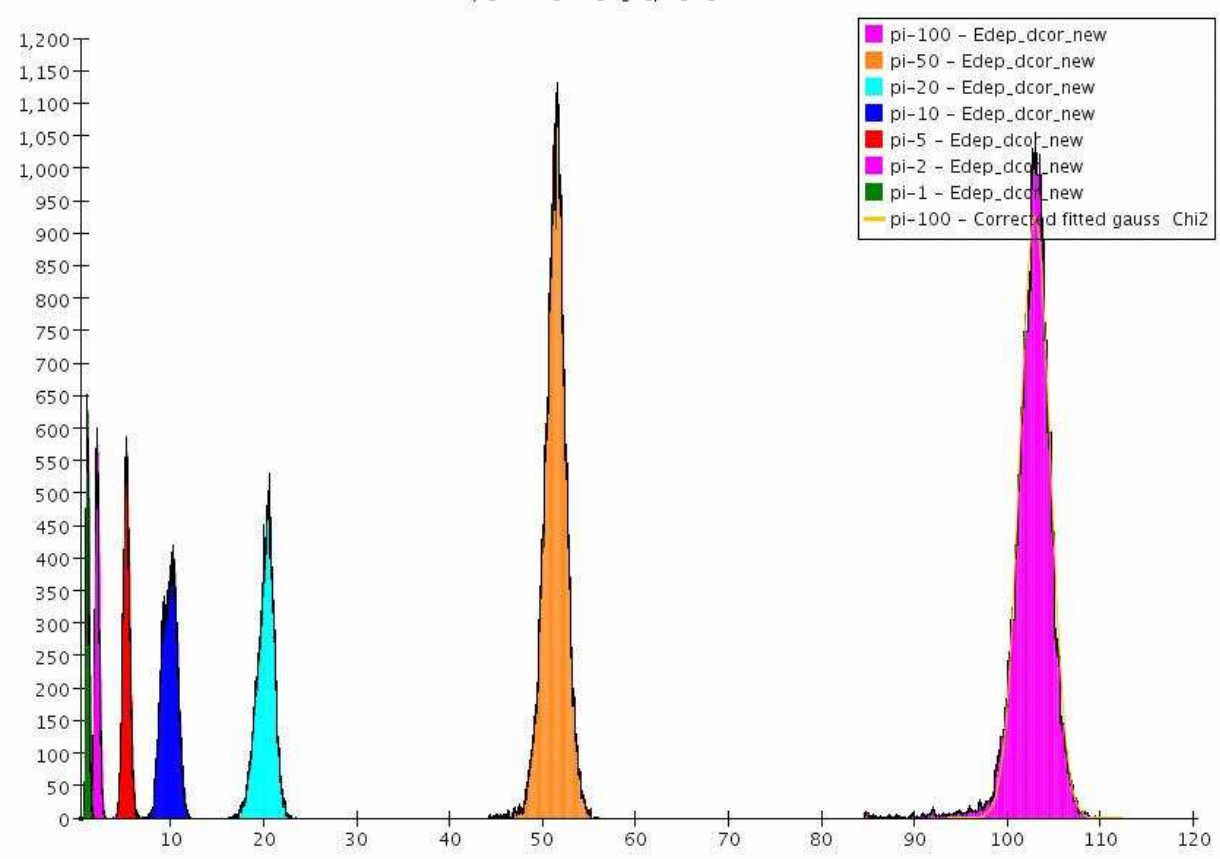

FIG. 20: D15 Dual Readout corrected $\pi^{-}$energy, from Hans Wenzel (compare with FIG. 21 for BGO)

LCPhys_digis_pi-_all_resol.aida - Edep_dcor_new

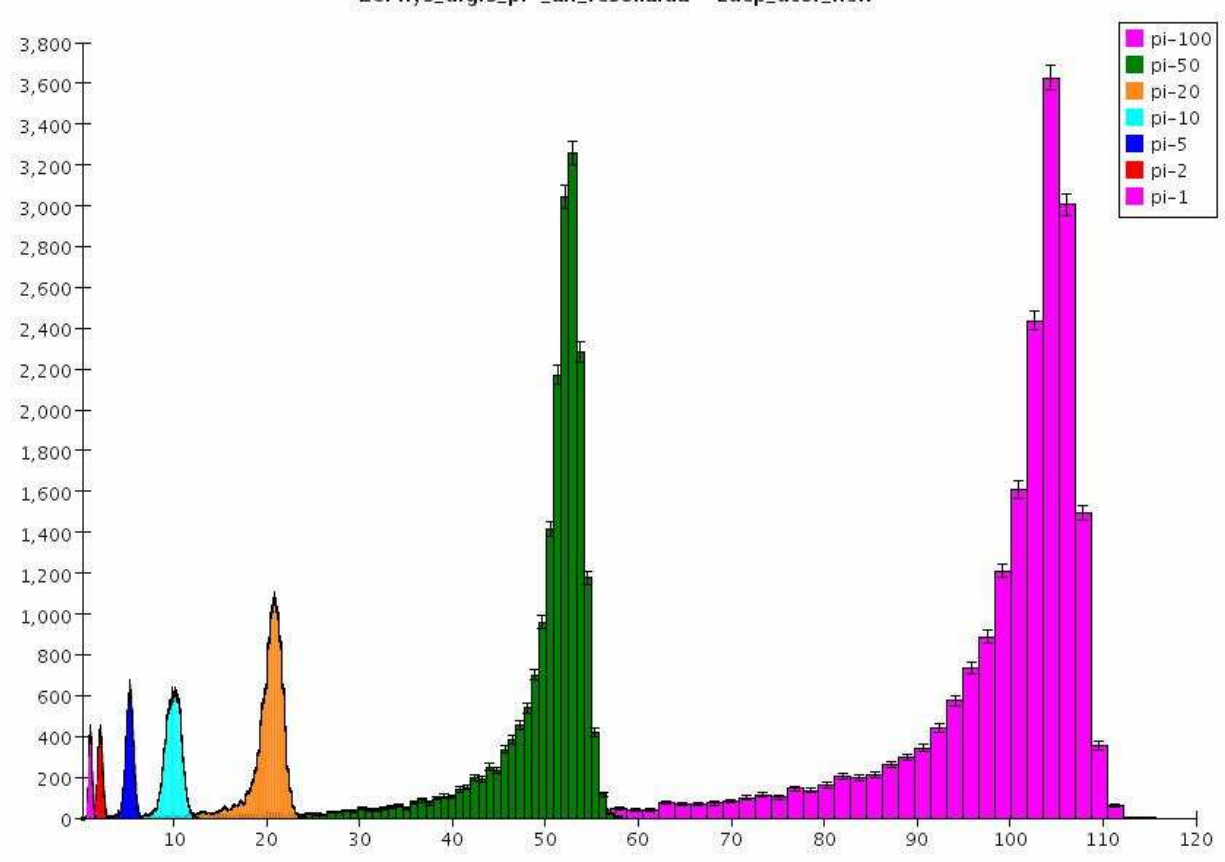

FIG. 21: BGO Dual Readout corrected $\pi^{-}$energy, from Hans Wenzel (compare with FIG. 20 for d15) 


\section{APPENDIX C: INTRODUCTORY COMPUTING INFO}

To use SLIC, jas3 to study Dual Readout Crystal calorimeter :

http://confluence.slac.stanford.edu/display/ilc/SLIC+Dual+Read+Out+Tutorial

LCSim Tutorials :

https://confluence.slac.stanford.edu/display/ilc/LCSim+Tutorials

To use OSG Grid :

http://confluence.slac.stanford.edu/display/ilc/How+do+I+use+the+OSG+Grid

Obtain DOE Grid Certificate :

http://security.fnal.gov/pki/Get-Personal-DOEGrids-Cert.html

ILC VO Registration : http://cd-amr.fnal.gov/ilc/ilcsim/ilcvo-registration.shtml

Setting up Netbeans : https://confluence.slac.stanford.edu/display/ilc/Setting+Up+Netbeans $+6.5+$ with + Maven +2 


\section{APPENDIX D: ANALYSIS FRAMEWORK}

This study is being performed on the ilcsim.fnal.gov computing cluster provided by the Fermilab Computing Division SSE Group. The analysis system is the Java Analysis Studio JAS3 http://jas.freehep.org/jas3/ which is a general purpose, open-source, data analysis tool.

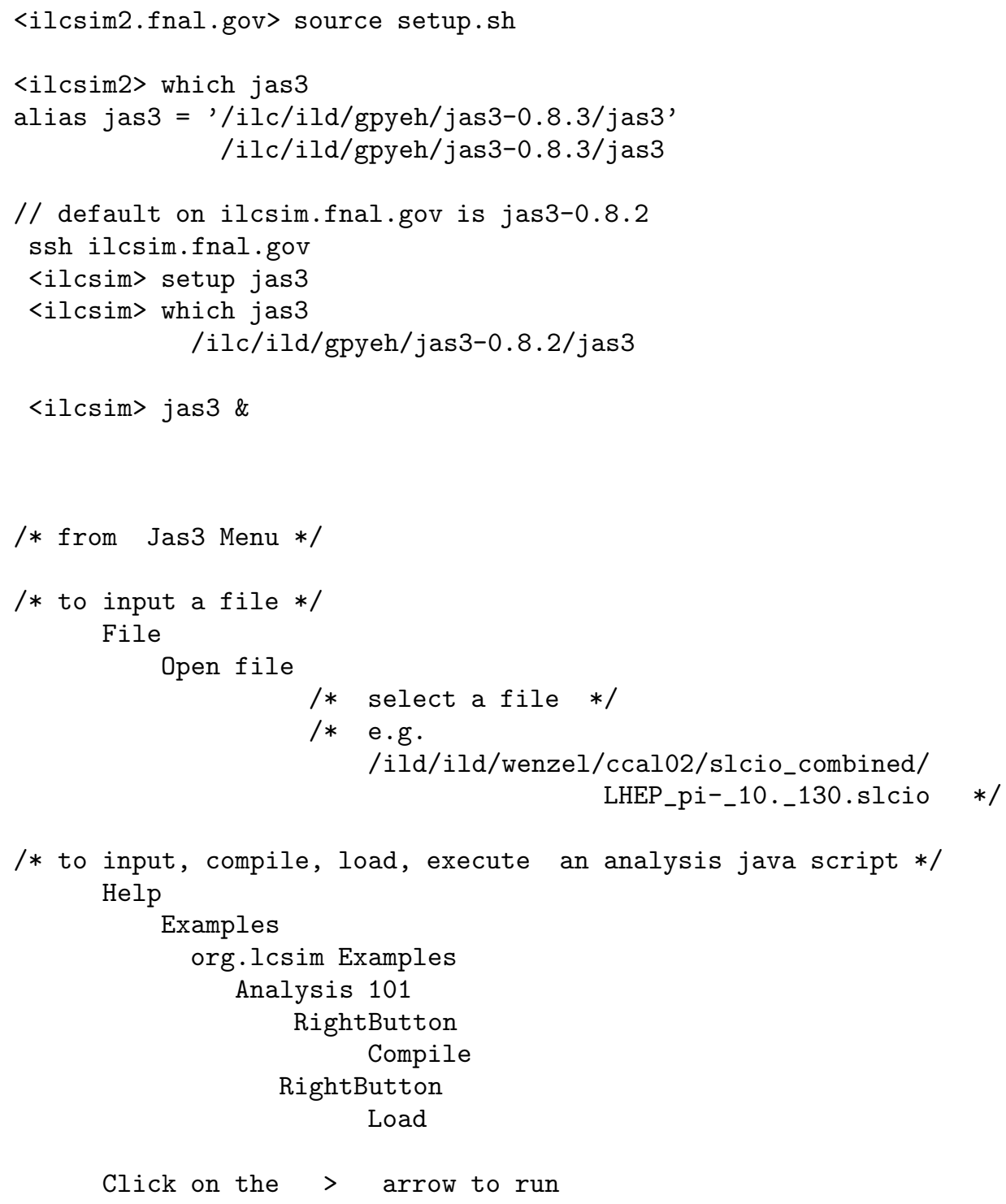


- New simulation files for density \& leakage study

<ilcsim2.fnal.gov>cd /ilc/ild/gpyeh/ccal02/slcio_combined <ilcsim2.fnal.gov> ls -lh *.slcio

\begin{tabular}{|c|c|c|c|}
\hline pyeh & $3.9 \mathrm{G}$ Nov & $3017: 16$ & BERT_-W_0_100GeV_25_15.slcio \\
\hline gpyeh & 97M Dec & $1215: 13$ & concat_events.slcio \\
\hline gpyeh & $112 \mathrm{M}$ Nov & $1712: 26$ & LCPhys_ccal02_25_15_e-_10.slcio \\
\hline gpyeh & $157 \mathrm{M}$ & $2314: 32$ & LCPhys_ccal02_25_15_e-_20.slcio \\
\hline gpyeh & $251 \mathrm{M}$ & $1717: 53$ & LCPhys_ccal02_25_15_e-_50.slcio \\
\hline pyeh & $3.1 \mathrm{G}$ & $1712: 45$ & LCPhys_ccal02_25_15_pi-_100.slcio \\
\hline byeh & 683M & $1712: 30$ & LCPhys_ccal02_25_15_pi-_10.slcio \\
\hline pyeh & $1.1 \mathrm{G}$ & $1717: 45$ & LCPhys_ccal02_25_15_pi-_20.slcio \\
\hline gpyeh & $2.0 \mathrm{G}$ & $1712: 38$ & LCPhys_ccal02_25_15_pi-_50.slcio \\
\hline gpyeh & $113 \mathrm{M}$ & $2411: 51$ & LCPhys_ccal02_75_45_e-_10.slcio \\
\hline gpyeh & $158 \mathrm{M}$ & $2515: 25$ & LCPhys_ccal02_75_45_e-_20.slcio \\
\hline gpyeh & 254M Nov & $2515: 22$ & LCPhys_ccal02_75_45_e-_50.slcio \\
\hline spyeh & $2.7 \mathrm{G}$ Nov & $2411: 36$ & LCPhys_ccal02_75_45_pi-_100.slcio \\
\hline & 694M & $2515: 06$ & LCPhys_ccal02_75_45_pi-_10.slcio \\
\hline pyeh & $1.1 \mathrm{G}$ & $2411: 47$ & LCPhys_ccal02_75_45_pi-_20.slcio \\
\hline pyeh & $2.0 \mathrm{G}$ & $2515: 16$ & LCPhys_ccal02_75_45_pi-_50.slcio \\
\hline gpyeh & $3.4 \mathrm{G}$ & $1314: 02$ & LCPhys_d10_50_30_pi-_100.slcio \\
\hline gpyeh & $712 \mathrm{M}$ & $13: 52$ & LCPhys_d10_50_30_pi-_10.slcio \\
\hline gpyeh & 5.4G Dec & $1417: 15$ & LCPhys_d10_50_30_pi-_200.slcio \\
\hline gpyeh & 1.1G Dec & $1313: 59$ & LCPhys_d10_50_30_pi-_20.slcio \\
\hline gpyeh & $2.1 \mathrm{G} \mathrm{I}$ & $1313: 50$ & LCPhys_d10_50_30_pi-_50.slcio \\
\hline spyeh & $3.3 \mathrm{G} \mathrm{I}$ & $2922: 02$ & LCPhys_d15_25_15_pi-_100.slcio \\
\hline spyeh & $680 \mathrm{M}$ & $3013: 54$ & LCPhys_d15_25_15_pi-_10.slcio \\
\hline gpyeh & $1.1 \mathrm{G}$ & $3013: 48$ & LCPhys_d15_25_15_pi-_20.slcio \\
\hline gpyeh & $2.1 \mathrm{G}$ & $13: 41$ & LCPhys_d15_25_15_pi-_50.slcio \\
\hline gpyeh & 52M Dec & $1215: 25$ & LCPhys_d15_50_30_e-_10.slcio \\
\hline gpyeh & 133M Dec & $1215: 26$ & LCPhys_d15_50_30_e-_50.slcio \\
\hline gpyeh & $3.3 \mathrm{G} \mathrm{I}$ & $212: 07$ & LCPhys_d15_50_30_pi-_100.slcio \\
\hline gpyeh & $683 \mathrm{M} \mathrm{I}$ & $13: 56$ & LCPhys_d15_50_30_pi-_10.slcio \\
\hline gpyeh & $5.2 \mathrm{G}$ & $11: 30$ & LCPhys_d15_50_30_pi-_200.slcio \\
\hline gpyeh & $1.1 \mathrm{G}$ & $13: 57$ & LCPhys_d15_50_30_pi-_20.slcio \\
\hline gpyeh & $2.1 \mathrm{G}$ & $: 12$ & LCPhys_d15_50_30_pi-_50.slcio \\
\hline gpyeh & 3.3G Nov & $3012: 30$ & LCPhys_d15_75_45_pi-_100.slcio \\
\hline gpyeh & $680 \mathrm{M}$ Nov & $3014: 25$ & LCPhys_d15_75_45_pi-_10.slcio \\
\hline spyeh & 1.1G Nov & $3013: 51$ & LCPhys_d15_75_45_pi-_20.slcio \\
\hline gpyeh & $2.1 \mathrm{G}$ & $3013: 11$ & LCPhys_d15_75_45_pi-_50.slcio \\
\hline gpyeh & $3.0 \mathrm{G}$ & $21: 10$ & LCPhys_d20_25_15_pi-_100.slcio \\
\hline gpyeh & $631 \mathrm{M}$ & $2411: 58$ & LCPhys_d20_25_15_pi-_10.slcio \\
\hline gpyeh & $985 \mathrm{M}$ & $2514: 04$ & LCPhys_d20_25_15_pi-_20.slcio \\
\hline gpyeh & $1.9 \mathrm{G}$ & $2514: 09$ & LCPhys_d20_25_15_pi-_50.slcio \\
\hline gpyeh & 3.OG Nov & $3015: 31$ & LCPhys_d20_50_30_pi-_100.slcio \\
\hline gpyeh & $633 \mathrm{M} \mathrm{Nov}$ & $3015: 35$ & LCPhys_d20_50_30_pi-_10.slcio \\
\hline pyeh & 4.8G Dec & $311: 34$ & LCPhys_d20_50_30_pi-_200.slcio \\
\hline gpyeh & 987M Nov & $3015: 34$ & LCPhys_d20_50_30_pi-_20.slcio \\
\hline gpyeh & $1.9 \mathrm{G} \mathrm{Nov}$ & $15: 33$ & LCPhys_d20_50_30_pi-_50.slcio \\
\hline gpyeh & 3.OG Nov & $3015: 20$ & LCPhys_d20_75_45_pi-_100.slcio \\
\hline gpyeh & $632 \mathrm{M}$ Nov & $3014: 40$ & LCPhys_d20_75_45_pi-_10.slcio \\
\hline gpyeh & 989M Nov & $3014: 42$ & LCPhys_d20_75_45_pi-_20.slcio \\
\hline gpyeh & $1.9 \mathrm{G} \mathrm{Nov}$ & $3015: 18$ & LCPhys_d20_75_45_pi-_50.slcio \\
\hline pyen & 114M Dec & $1215: 22$ & LCPhys_d7_50_30_e-_10.slcio \\
\hline gpyeh & 254M Dec & $1215: 20$ & LCPhys_d7_50_30_e-_50.slcio \\
\hline & & & LCPhys_d7_50_30_pi-_100.slcic \\
\hline
\end{tabular}




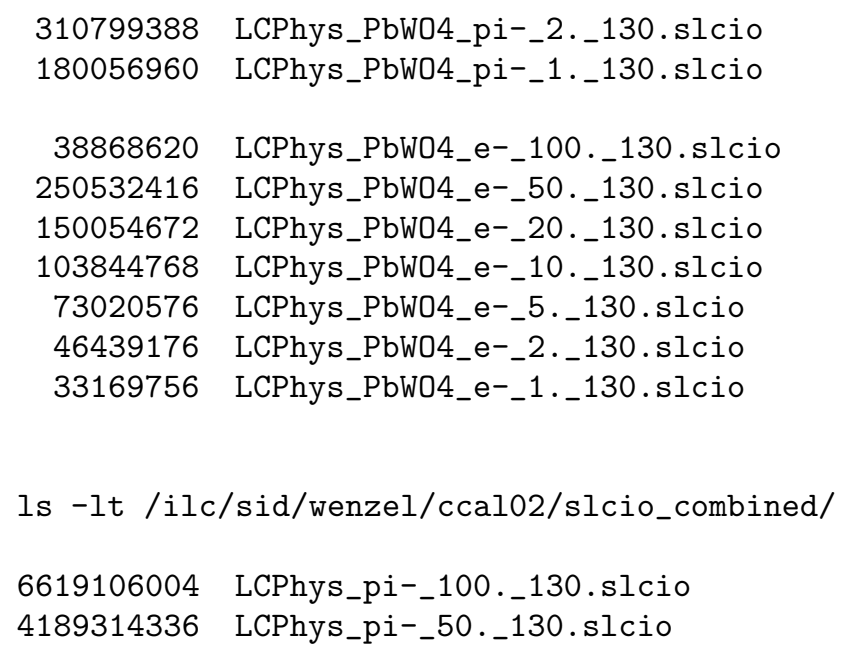

- Previously already available simulation files

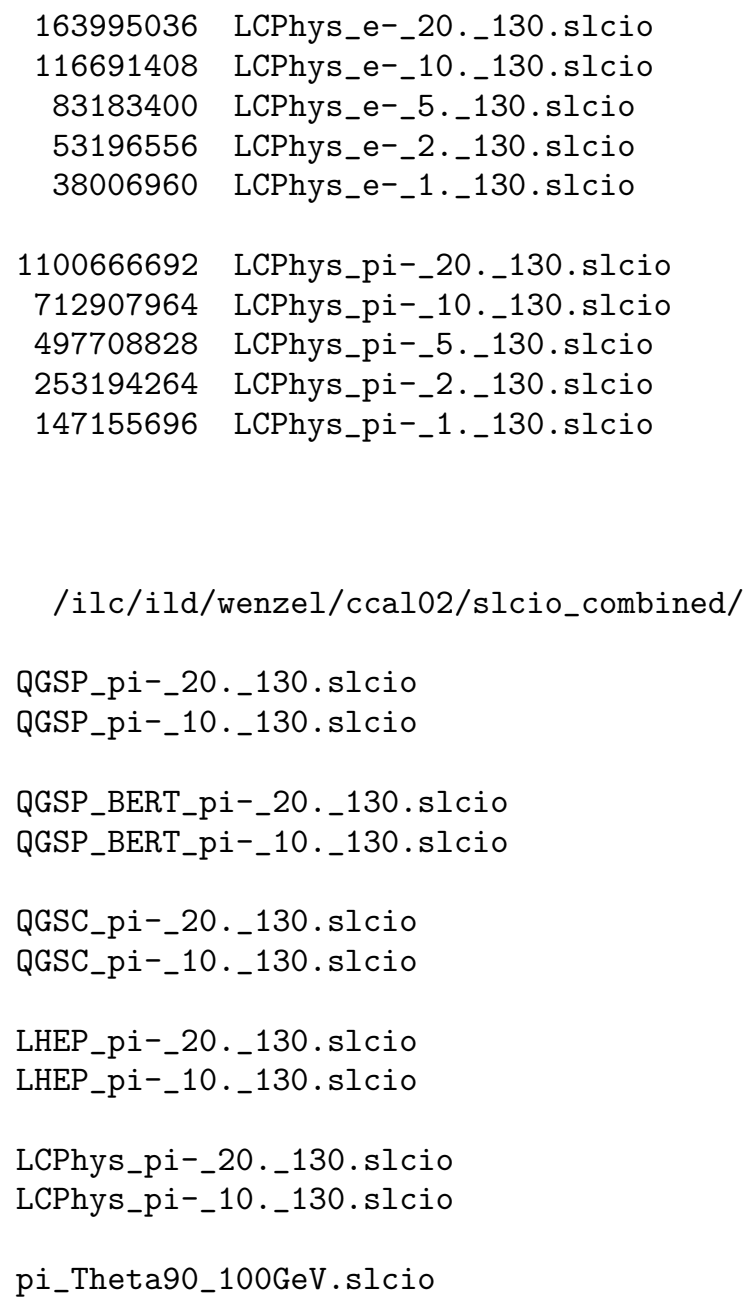




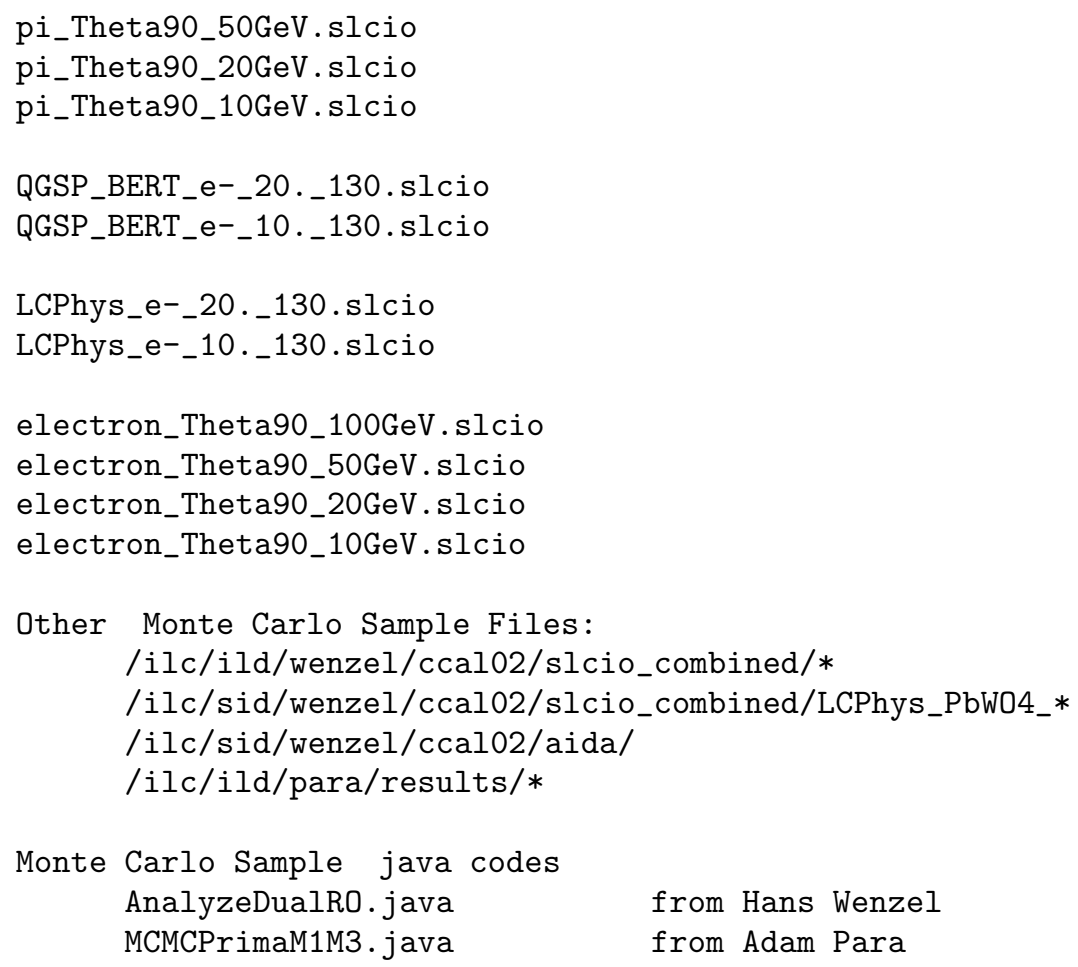


- stdhep files to generate $\mathrm{W}$ or $\mathrm{Z}$ events

<ilcsim.fnal.gov> ls -lt /ilc/ild/wenzel/ccal02/stdhep/Z0_*.stdhep

W_*.stdhep

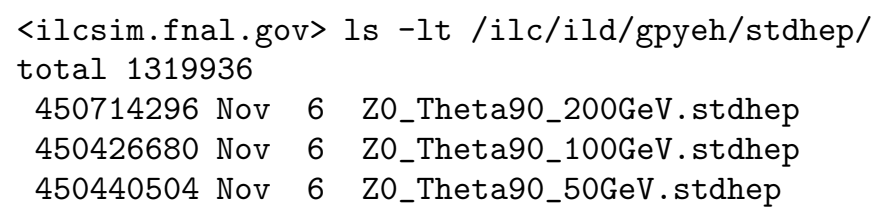


- aida files

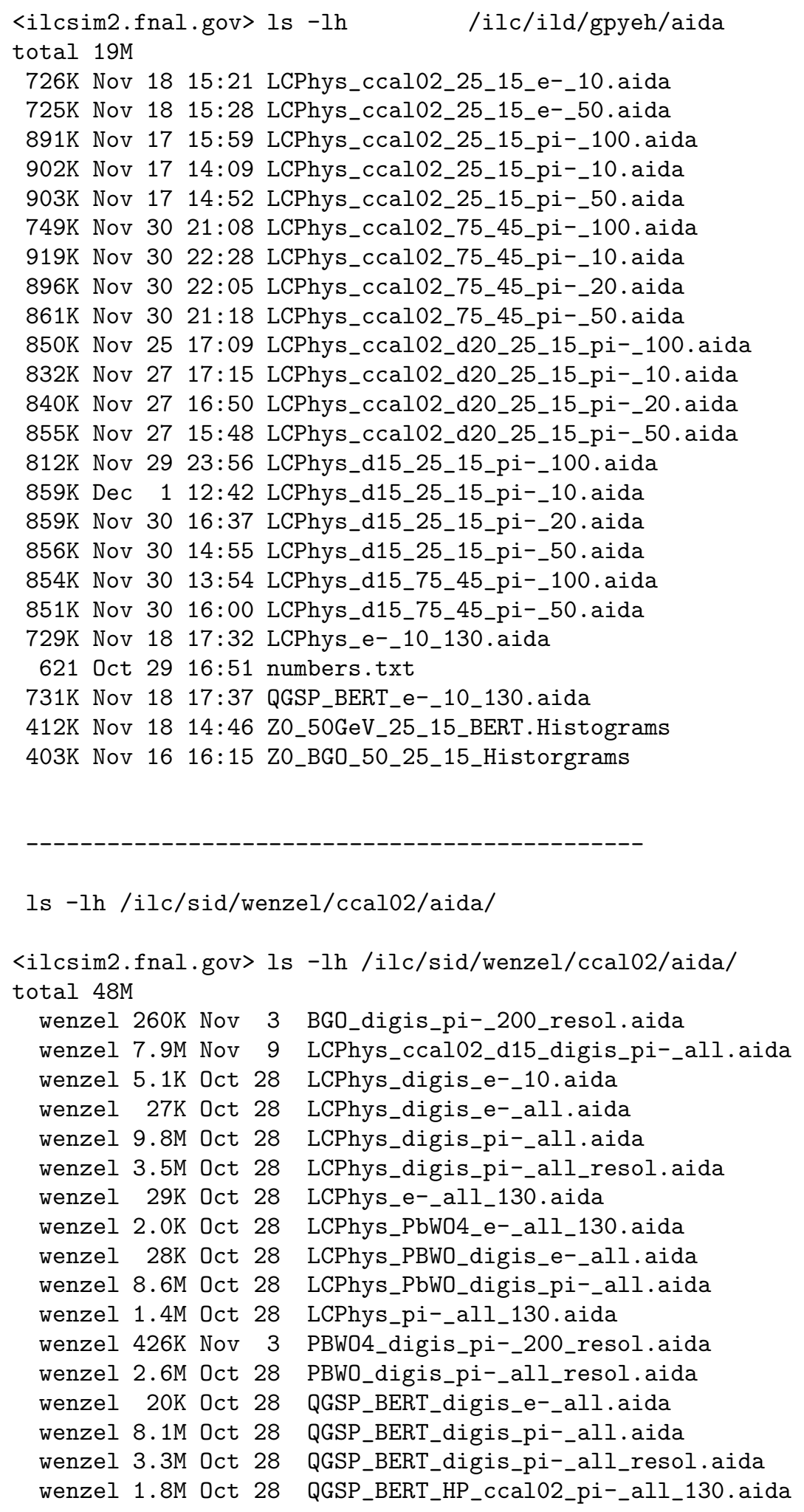




\section{APPENDIX E: SIMULATION MODIFICATION FROM BGO TO PBWO4}

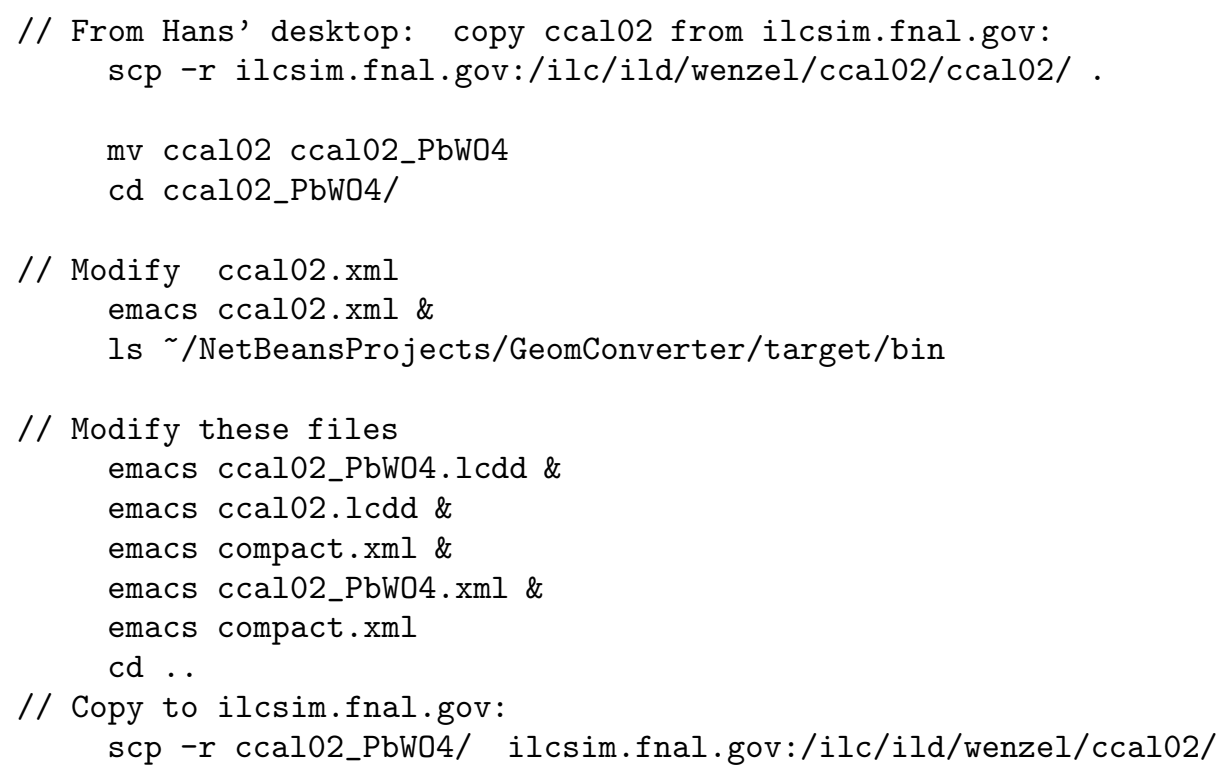




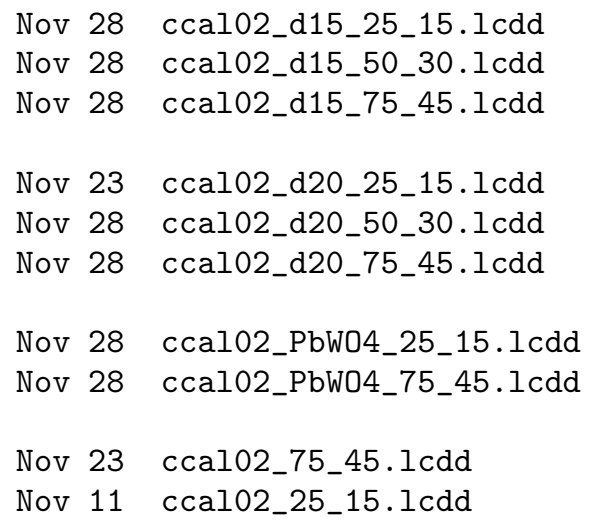

.zip must be copied to the $/$.lcsim/cache for jas and lcsim to pick it up.

cp http $\% 3 \mathrm{~A} \% 2 \mathrm{~F} \% 2 \mathrm{Fwww} .1 \mathrm{csim}$. org $\% 2 \mathrm{Fdetectors} \% 2 \mathrm{Fccal02}$ _d 15. zip $~ / .1 c s i m /$ cache

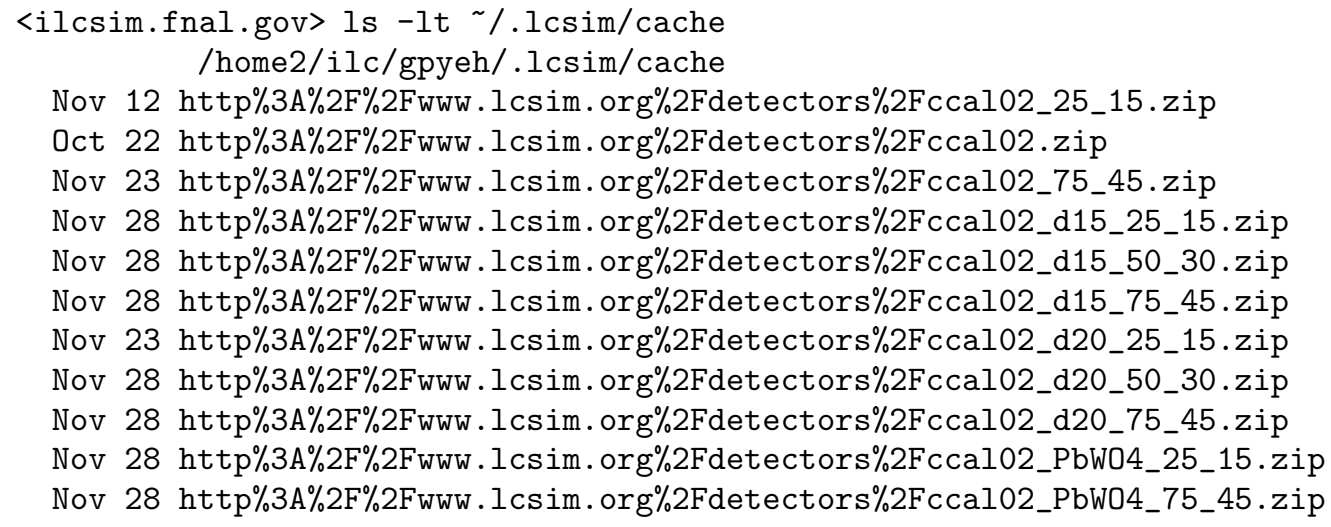

get $\$$ \{detector\}.lcdd and the corresponding zip file.

The segmentation in EM and Had sections is in transverse plane only.

Units are $\mathrm{mm}$. Bfield in tesla.

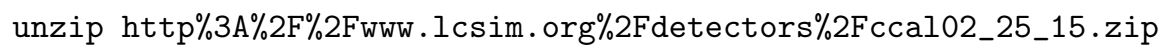

<ilcsim.fnal.gov> ls -lt/ilc/ild/gpyeh/grid_gp_d15

Nov 17 slic_grid_Z0_50GeV_25_15_BERT.run

Nov 17 slic_grid_Z0_50GeV_25_15_BERT.csh

Nov 12 slic_grid_Z0_50GeV_25_15.run 


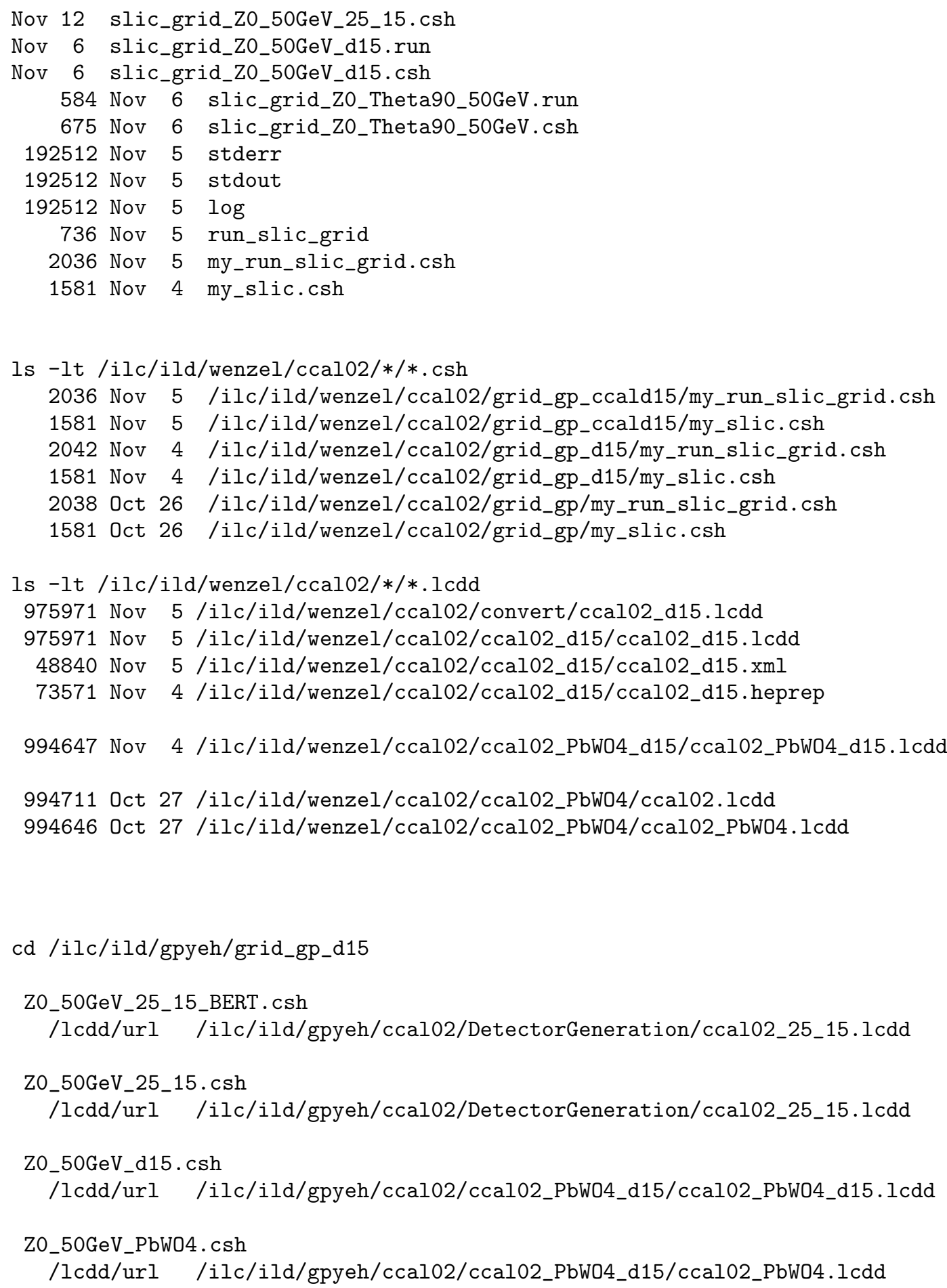




\section{APPENDIX F: USING OSG GRID}

// DOE Grid Certificate

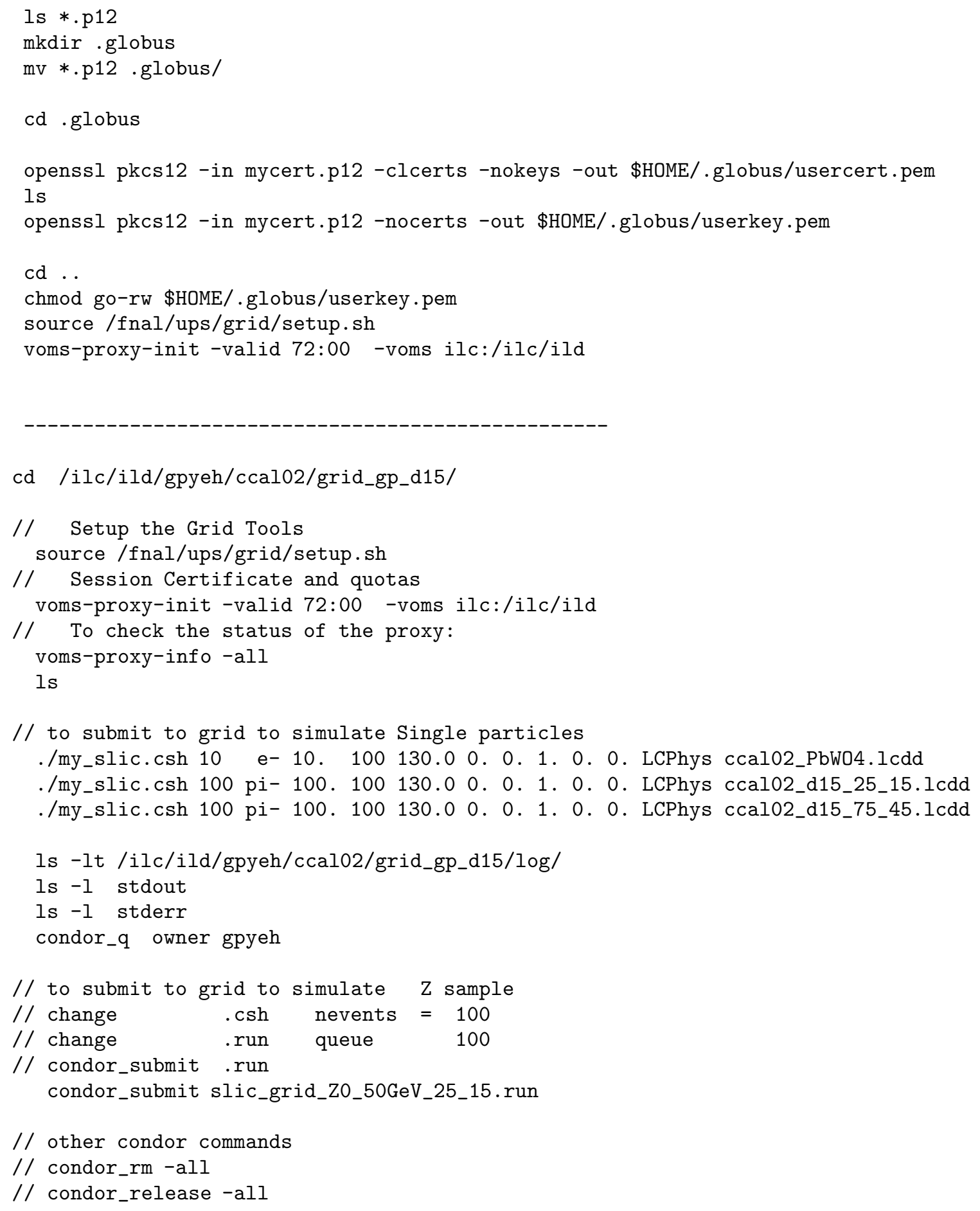


Date: Tuesday, Dec. 1, 2009

From: Gratia Operation <gratia-operation@opensciencegrid.org>

OSG metrics for VO ILC for November, 2009-11-01 to 2009-11-30

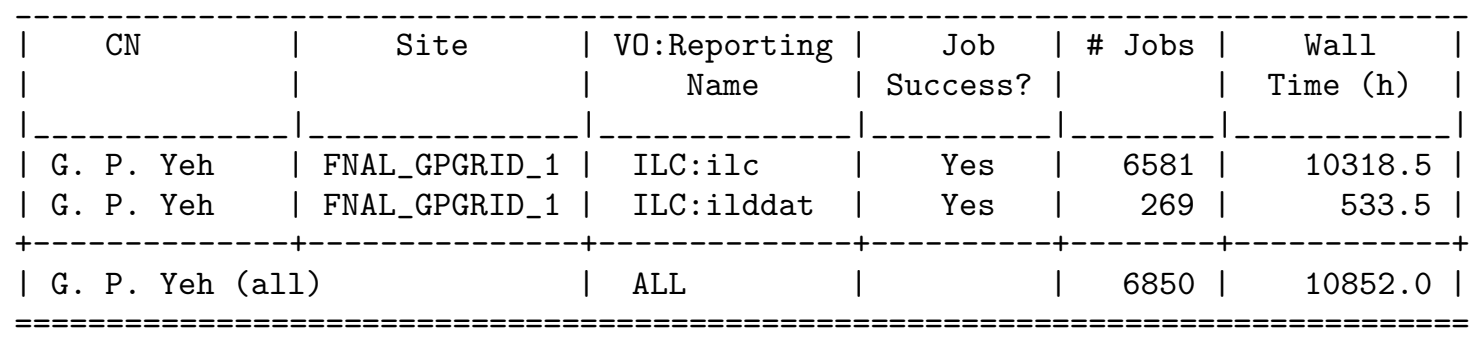

OSG metrics for VO ILC for December, 2009-12-01 to 2009-12-31

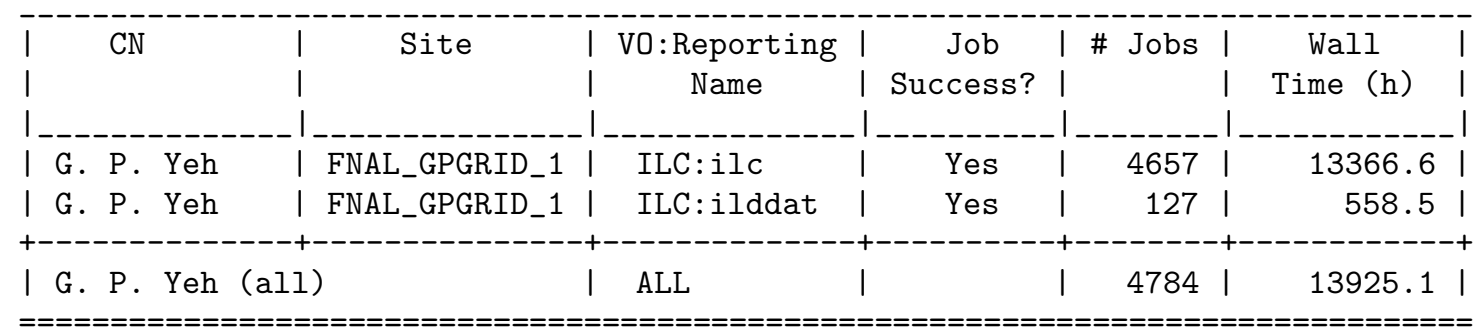

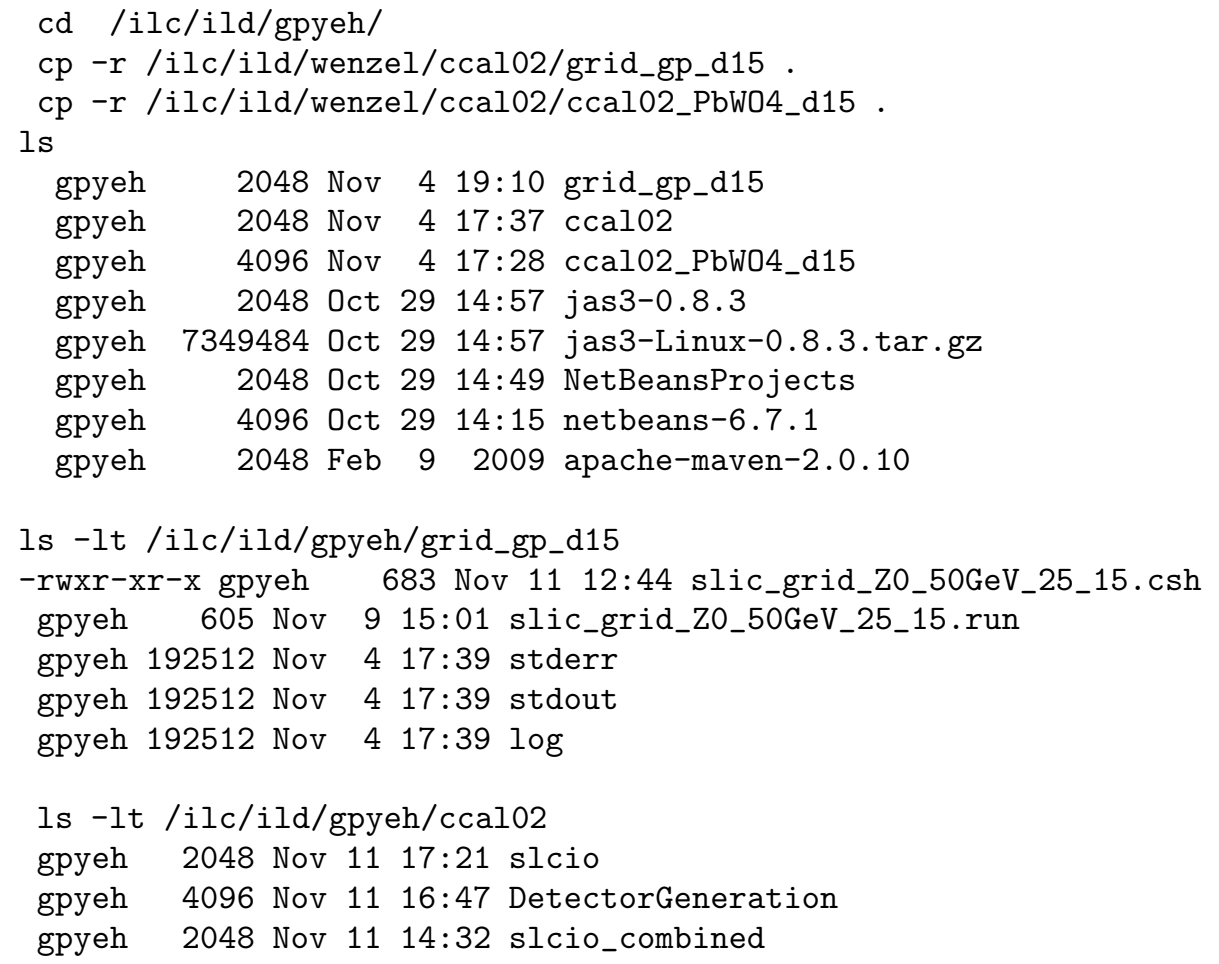




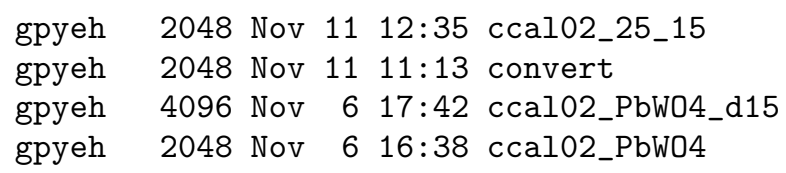

// lcio concat combine slcio files

cd /ilc/ild/gpyeh/ccal02/slcio_combined/

cd /grid/app/CherSimDist_may2009/SimDist/packages/lcio/HEAD/

source setup.sh

// which lcio

cd -

pwd

/ilc/ild/gpyeh/ccal02/slcio_combined/

Is -1/ilc/ild/gpyeh/ccal02/slcio/LCPhys_ccal02_25_15.1cdd_e-*.slcio > combine_e10.txt

ls -1 /ilc/ild/gpyeh/ccal02/slcio/LCPhys_ccal02_25_15.1cdd_pi-_10.*.slcio > combine_pi10.txt

// the following gets /bin/sh error on ilcsim.fnal.gov

It works on ilcsim2.fnal.gov

lcio concat -i combine.txt -o Z0_50GeV_25_15.slcio

lcio concat -i combine_d15_25_15_pi100.txt -o LCPhys_d15_25_15_pi-_100.slcio

Is $-1 \mathrm{~h}$

gpyeh 3319780264 Nov 17 LCPhys_ccal02_25_15_pi-_100.slcio

gpyeh 2098802104 Nov 17 LCPhys_ccal02_25_15_pi-_50.slcio

gpyeh 715474196 Nov 17 LCPhys_ccal02_25_15_pi-_10.slcio

gpyeh 116763700 Nov 17 LCPhys_ccal02_25_15_e-_10.slcio

gpyeh $\quad 7990$ Nov 17 combine_pi50.txt

gpyeh $\quad 8090$ Nov 17 combine_pi100.txt

gpyeh $\quad 7990$ Nov 17 combine_pi10.txt

gpyeh $\quad 7890$ Nov 17 combine_e10.txt

gpyeh 27368648 Nov 12 Z0_50GeV_25_15_s.slcio

gpyeh 255 Nov 12 combine.txt

ls - lh $\ldots /$ slcio

rm -f /ilc/ild/gpyeh/ccal02/slcio/LCPhys_ccal02_25_15.lcdd_pi-_10.*.slcio 